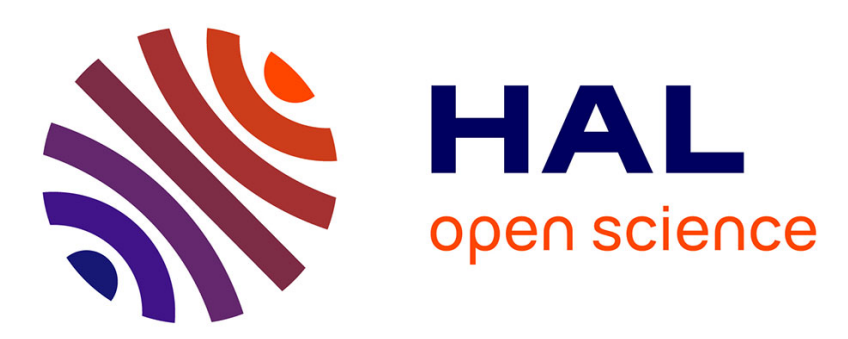

\title{
Feuilletages singuliers de codimension un, Groupoïde de Galois et intégrales premières \\ Guy Casale
}

\section{To cite this version:}

Guy Casale. Feuilletages singuliers de codimension un, Groupoïde de Galois et intégrales premières. Annales de l'Institut Fourier, 2006, 56 (3), pp.735-779. 10.5802/aif.2198 . hal-00004495

\section{HAL Id: hal-00004495 \\ https://hal.science/hal-00004495}

Submitted on 17 Mar 2005

HAL is a multi-disciplinary open access archive for the deposit and dissemination of scientific research documents, whether they are published or not. The documents may come from teaching and research institutions in France or abroad, or from public or private research centers.
L'archive ouverte pluridisciplinaire $\mathbf{H A L}$, est destinée au dépôt et à la diffusion de documents scientifiques de niveau recherche, publiés ou non, émanant des établissements d'enseignement et de recherche français ou étrangers, des laboratoires publics ou privés. 


\title{
FEUILLETAGES SINGULIERS DE CODIMENSION UN, GROUPOÏDE DE GALOIS ET INTÉGRALES PREMIĖRES
}

\author{
GUY CASALE
}

\section{TABLE DES MATIÈRES}

Introduction $\quad 1$

1. Définitions et rappels 3

2. Le groupoïde de Galois d'un feuilletage 10

3. Groupoïdes de Galois et Suites de Godbillon-Vey 11

4. Groupoïdes de Galois et intégrales premières 19

5. Le groupoïde de Galois d'un germe de feuilletage de $\left(\mathbb{C}^{2}, 0\right)$ à singularité réduite 24

6. Groupoïdes de Galois et extensions fortement normales 27

Références 33

\section{INTRODUCTION}

En 2001, B. Malgrange a proposé dans Le groupö̈de de Galois d'un feuilletage ([Ma4]) une manière de généraliser le groupe de Galois différentiel, défini pour une équation différentielle linéaire, aux feuilletages singuliers. Deux changements importants par rapport aux théories précédentes apparaissent. Premièrement, on perd la structure de groupe algébrique remplacée par celle de $\mathcal{D}$-groupoïde de Lie. Deuxièmement, alors que le groupe de Galois agissait sur les variables dépendantes (les inconnues des équations différentielles), le groupoïde de Galois agit sur l'ensemble des variables dépendantes et indépendantes (l'espace portant le feuilletage donné par les équations différentielles).

Le groupoïde de Galois est défini par le système maximal d'équations aux dérivées partielles qui vérifie les conditions suivantes :

- Les flots des champs de vecteurs tangents au feuilletage sont des solutions de ce système.

- Les inclusions de la définition 1.2 sont vérifiées. Ces dernières signifient que l'identité est solution du système, que la composée de deux solutions est une solution et que l'inverse d'une solution est encore une solution.

D'après [Ma4], un système d'équations vérifiant ce dernier point est appelé $\mathcal{D}$-groupoïde de Lie.

Des définitions analogues bien qu'imprécises ont été esquissées par J. Drach [Dr1] et E. Vessiot [Ves1], [Ves2]. Elles étaient basées sur la notion de systèmes automorphes d'intégrales premières du feuilletage. Les preuves de l'existence de tels systèmes semblent, malheureusement, incomplètes. En basant sa définition sur les propriétés dynamiques du feuilletage, sans aucune référence aux intégrales premières, et en utilisant son théorème d'involutivité générique [Ma2], B. Malgrange contourne les problèmes de définition et résoud les problèmes d'existence. 
Dans cet article, nous étudierons le groupoïde de Galois d'un germe de feuilletage de codimension un. Nous rappellerons dans une première partie les définitions locales de $\mathcal{D}$-groupoïde de Lie [Ma4] et les résultats relatifs aux $\mathcal{D}$-groupoïde de Lie au-dessus d'un disque de $\mathbb{C}$ ([Cas2], [Ma3]). Ces derniers nous permettrons, dans une deuxième partie, de préciser la nature du système d'équations aux dérivées partielles définissant le groupoïde de Galois du feuilletage et de caractériser sa taille par un nombre : le rang transverse du groupoïde de Galois du feuilletage. Ce nombre appartient à $\{0,1,2,3, \infty\}$.

Dans la troisième partie, nous montrerons les liens entre le groupoïde de Galois et l'existence de structures transverses. Nous discuterons suivant le rang transverse du groupoïde de Galois l'existence de structures méromorphes transverses euclidienne (rang transverse égale à un), affine (rang transverse égale à deux) ou projective (rang transverse égale à trois). Nous énoncerons le résultat en terme de suites de Godbillon-Vey méromorphes pour le feuilletage, associées aux structures transverses. Le théorème 3.2 donne l'égalité du rang transverse du groupoïde de Galois avec la longueur minimale des suites de Godbillon-Vey méromorphes du feuilletage dans le cas des suites de longueur 1, 2 ou 3. Ces résultats ont été annoncés dans [Ma5] où B. Malgrange prouve une des deux inégalités d'une manière plus géométrique mais essentiellement analogue à la notre. La preuve que nous donnons de l'autre inégalité semble être différente de celle de B. Malgrange.

Dans [Cas2] nous caractérisons les germes de difféomorphismes de $(\mathbb{C}, 0)$ solutions d'un $\mathcal{D}$-groupoïde de Lie au-dessus de $(\mathbb{C}, 0)$. Les germes de feuilletages de $\left(\mathbb{C}^{2}, 0\right)$ à singularités réduites étant complètement décrit par leurs holonomies, nous étudierons plus pariculièrement ces feuilletage dans la quatrième partie. Nous expliquerons comment les résultats de la partie précédente complétés par ceux de [Cas2] redonne la caractérisation en terme d'invariants analytiques des germes de feuilletages de $\left(\mathbb{C}^{2}, 0\right)$ à singularités réduites admettant une structure transverse méromorphe affine ou projective. Nous retrouvons ainsi de manière "galoisienne" les résultats de M. Berthier et F. Touzet [B-T] et ceux de F. Touzet [Tou2].

Dans la cinquième partie, nous discuterons des différents types de transcendance d'intégrales premières (définition 4.1) du feuilletage caractérisés par le groupoïde de Galois (théorème 4.2). Suivant le rang transverse du groupoïde de Galois, le feuilletage admet une intégrale première de type méromorphe, Darboux, Liouville ou Riccati. Dans le cas d'un feuilletage dont le groupoïde de Galois est de rang transverse deux ou trois, le résultat provient essentiellement d'un théorème de M. Singer [Si] et de sa version projective [Cas1] via le théorème 3.2. Pour les feuilletages de groupoïde de Galois de rang transverse un, la démonstration consiste à prouver une version adaptée du théorème de Singer. Le cas des feuilletages de groupoïde de Galois de rang transverse nul se traite d'un manière différente, nous construirons un quotient de l'espace des feuilles naturellement muni d'une structure de courbe analytique.

Dans la dernière partie, nous décrirons quelques-unes des relations entre le groupoïde de Galois d'un feuilletage et la notion d'extension fortement normale de E. R. Kolchin ([Ko]). Les intégrales premières construites dans la partie précédente sont naturellement des éléments d'une extension fortement normale du corps des fonctions méromorphes. Nous prouvons ensuite la réciproque : si il existe une intégrale première du feuilletage dans une extension fortement normale du corps des fonctions méromorphes, le groupoïde de Galois est de rang transverse fini (théorème 6.4). 


\section{DÉfinitions et RAPPELS}

Dans [Ma4] B. Malgrange définit la notion de $\mathcal{D}$-groupoïde de Lie et montre plusieurs propriétés de ces objets. Nous commençons par rappeler les définitions relatives aux $D$ groupoïdes de Lie au-dessus d'un polydisque $\Delta$ de $\mathbb{C}^{n}$.

L'espace des jets d'ordre $k$ d'applications inversibles de $\Delta$ dans $\Delta$ sera noté $J_{k}^{*}(\Delta)$. Le choix d'une coordonnée $x$ sur $\Delta$ permet de faire l'identification :

$$
J_{k}^{*}(\Delta)=\Delta \times \Delta \times G L_{n}(\mathbb{C}) \underset{2 \leq|\alpha| \leq k}{\times} \mathbb{C}^{n|\alpha|}
$$

avec les coordonnées naturelles $\left(x_{i}, y_{i}, y_{i}^{\alpha}\right)$. Le multi-indice $\alpha$ appartient à $\mathbb{N}^{n}$ et on note $|\alpha|$ la somme de ses composantes. Nous noterons $\epsilon_{j}$ le multi-indice dont la seule coordonnée non nulle est la $j$-ième et est égale à 1 . On munit l'espace $\Delta \times \Delta$ du faisceau d'anneaux

$$
\mathcal{O}_{J_{k}^{*}(\Delta)}=\mathcal{O}_{\Delta \times \Delta}\left[y_{i}^{\alpha}, \frac{1}{\operatorname{det}\left(y_{i}^{\epsilon_{j}}\right)}\right]
$$

qui s'identifie à l'anneau des équations aux dérivées partielles, polynomiales en les dérivées, d'ordre inférieur ou égal à $k$ ayant $n$ variables indépendantes et $n$ variables dépendantes. Étant donné un jet d'application $y(x)$ de $\Delta$ dans $\Delta$, nous regrouperons les dérivées $y_{i}^{\alpha}$ suivant leurs ordres $|\alpha|$. Nous noterons $y^{\prime}$ la jacobienne de $y$ par rapport à $x, y^{\prime \prime}$ la hessienne (qui est élément de $S^{2} \mathbb{C}^{n} \otimes \mathbb{C}^{n}$ ) de $y$ par rapport à $x, y^{\prime \prime \prime}$ élément de $S^{3} \mathbb{C}^{n} \otimes \mathbb{C}^{n}$ la forme trilinéaire des dérivées troisièmes ...

Les espaces $J_{k}^{*}(\Delta)$ sont de plus munis d'une structure de groupoïde par la donnée

- de la projection source $s: J_{k}^{*}(\Delta) \rightarrow \Delta$ définie par $s(x, y, \ldots)=x$,

- de la projection but $t: J_{k}^{*}(\Delta) \rightarrow \Delta$ définie par $t(x, y, \ldots)=y$,

- d'une composition $c: J_{k}^{*}(\Delta) \times_{\Delta} J_{k}^{*}(\Delta) \rightarrow J_{k}^{*}(\Delta)$ définie sur les couples de jets $(h, g)$ tels que $t(h)=s(g)$ par

$$
c\left(\left(x, y, y^{\prime}, y^{\prime \prime}, \ldots\right),\left(y, z, z^{\prime}, z^{\prime \prime}, \ldots\right)\right)=\left(x, z, z^{\prime} y^{\prime}, z^{\prime \prime}\left(y^{\prime}, y^{\prime}\right)+z^{\prime} y^{\prime \prime}, \ldots\right),
$$

- d'une identité, la sous-variété définie par les équations $x_{i}=y_{i}$ et $y_{i}^{\epsilon_{j}}=\delta_{i}^{j}$ pour $0 \leq i, j \leq n$ et $y_{i}^{\alpha}=0$ pour $|\alpha| \geq 2$, donnée par le plongement $e: \Delta \rightarrow J_{k}^{*}(\Delta)$ par $e(x)=(x, x, i d, 0, \ldots, 0)$,

- d'une inversion $i: J_{k}^{*}(\Delta) \rightarrow J_{k}^{*}(\Delta)$ qui à un jet $\left(x, y, y^{\prime}, y^{\prime \prime}, \ldots\right)$ fait correspondre le jet

$$
\left(y, x,\left(y^{\prime}\right)^{-1},-\left(y^{\prime}\right)^{-1} y^{\prime \prime}\left(\left(y^{\prime}\right)^{-1},\left(y^{\prime}\right)^{-1}\right), \ldots\right),
$$

On a de plus $n$ dérivations $D_{i}: \mathcal{O}_{J_{k}^{*}(\Delta)} \rightarrow \mathcal{O}_{J_{k+1}^{*}(\Delta)}$ qui correspondent aux dérivations partielles de fonctions composées. Étant donnée une équation $E$ :

$$
D_{i} E=\frac{\partial E}{\partial x_{i}}+\sum_{\ell, \alpha} \frac{\partial E}{\partial y_{\ell}^{\alpha}} y_{\ell}^{\alpha+\epsilon_{i}} .
$$

Toutes ces flèches sont compatibles au projections naturelles $J_{k+1}^{*}(\Delta) \rightarrow J_{k}^{*}(\Delta)$ ce qui permet de les définir sur l'espace $\Delta \times \Delta$ muni de l'anneau $\mathcal{O}_{J^{*}(\Delta)}=\lim \mathcal{O}_{J_{k}^{*}(\Delta)}$. Les définitions suivantes sont issues de [Ma4].

Définition 1.1. Un groupoïde d'ordre $k$ sur $\Delta$ est donné par un idéal (= faisceau d'idéaux) cohérent $\mathcal{I}_{k}$ de $\mathcal{O}_{J_{k}^{*}(\Delta)}$ tel que :

(1) $\mathcal{I}_{k} \subset \operatorname{Ker}\left(e^{*}\right)$ 
(2) $i^{*} \mathcal{I}_{k} \subset \mathcal{I}_{k}$

(3) $c^{*} \mathcal{I}_{k} \subset \mathcal{I}_{k} \otimes_{\mathcal{O}_{\Delta}} 1+1 \otimes_{\mathcal{O}_{\Delta}} \mathcal{I}_{k}$ (la somme étant prise comme somme d'idéaux).

Cette définition est naturelle mais en pratique trop restrictive pour la définition de groupoïde de Galois telle qu'elle est donnée dans la suite. Il faut alors utiliser la définition plus souple suivante.

Définition 1.2. Un $\mathcal{D}$-groupoïde de Lie sur $\Delta$ est donné par un idéal réduit $\mathcal{I}$ de $\mathcal{O}_{J^{*}(\Delta)}$ tel que

- tous les idéaux $\mathcal{I}_{\ell}=\mathcal{I} \cap \mathcal{O}_{J_{\ell}^{*}(\Delta)}$ sont cohérents,

- I soit stable par dérivation,

- pour tout ouvert relativement compact $U \subset \Delta$ il existe un entier $k$ et un ensemble analytique fermé $Z$ dans $U$ tels que pour tout $\ell \geq k, \widetilde{\mathcal{I}}_{\ell}=\left.\mathcal{I}_{\ell}\right|_{U}$ vérifie

(i) les inclusions (1) et (2) de la définition 1.1,

(ii) l'inclusion (3) de la définition 1.1 sur tout voisinage de $(x, y, z) \in(U-Z) \times$ $(U-Z) \times(U-Z)$.

Dans cet article nous ne nous intéresserons qu'aux $\mathcal{D}$-groupoïdes de Lie au-dessus d'un polydisque de taille arbitrairement petite. En nous plaçant directement sur un polydisque plus petit que celui de définition, les points $(i)$ et $(i i)$ seront vérifiés sur tout le polydisque.

Une solution de $\mathcal{I}$ en $p \in \Delta$ est un morphisme $u$ de $\mathcal{O}_{J^{*}(\Delta)} / \mathcal{I}$ dans $\mathcal{O}_{\Delta, p}$ au-dessus de la restriction $\mathcal{O}_{\Delta} \rightarrow \mathcal{O}_{\Delta, p}$, tel que $u\left(D_{i} E\right)=\frac{\partial}{\partial x_{i}} u(E)$. En regardant $f=u(y)$, on obtient un germe $f:(\Delta, p) \rightarrow(\Delta, q)$ satisfaisant les équations différentielles de l'idéal $\mathcal{I}$. On définit de même les solutions formelles comme morphismes dans $\widehat{\mathcal{O}}_{\Delta, p}$. Réciproquement, un germe d'application inversible $f$, solution des équations différentielles engendrant $\mathcal{I}$ définit un morphisme $u$ par $u(y)=f$. Nous identifierons souvent, par abus de langage, un $\mathcal{D}$-groupoïde de Lie avec ses solutions formelles. En particulier nous dirons qu'un $\mathcal{D}$ groupoïde de Lie d'idéal $\mathcal{I}$ est inclus dans un second d'idéal $\mathcal{J}$ si l'idéal $\mathcal{I}$ contient l'idéal $\mathcal{J}$ et qu'un $\mathcal{D}$-groupoïde de Lie contient un difféomorphisme si ce dernier est solution des équations de $\mathcal{I}$.

Donnons quelques exemples de $\mathcal{D}$-groupoïdes de Lie :

Exemple 1.3. Le groupoïde d'invariance d'une fonction méromorphe $\frac{P}{Q}$ est un $\mathcal{D}$-groupoïde de Lie dont l'idéal est différentiablement engendré par

$$
Q(x) P(y)-Q(y) P(x) .
$$

Les propriétés (1) et (2) d'un $\mathcal{D}$-groupoïde de Lie sont évidentes. La propriété (3) est vraie en dehors du lieu d'indétermination de $\frac{P}{Q}$. En effet, les égalités

$$
\begin{aligned}
& Q(z)[Q(x) P(y)-Q(y) P(x)] \\
& \quad=Q(y)[Q(x) P(z)-Q(z) P(x)]-Q(x)[Q(z) P(y)-Q(y) P(z)]
\end{aligned}
$$

et

$$
\begin{aligned}
P(z)[Q(x) P(y)-Q(y) & P(x)] \\
& =P(y)[Q(x) P(z)-Q(z) P(x)]-P(x)[Q(z) P(y)-Q(y) P(z)]
\end{aligned}
$$

donnent l'inclusion voulue tant que $P(z) \neq 0$ ou $Q(z) \neq 0$. 
Exemple 1.4. Le groupö̈de d'invariance d'un champ méromorphe de tenseurs, T, est un $\mathcal{D}$-groupoïde de Lie dont l'idéal est différentiablement engendré par les composantes de $\Gamma^{*} T-T$. Les propriétés (1), (2) et (3) proviennent de l'égalité

$$
\Gamma_{2}^{*} \Gamma_{1}^{*} T-T=\Gamma_{2}^{*}\left(\Gamma_{1}^{*} T-T\right)-\left(\Gamma_{2}^{*} T-T\right)
$$

L'ensemble $Z$ est alors inclus dans le lieu des pôles du champ $T$.

Exemple 1.5. Le groupoïde d'invariance d'un champ d'hyperplan donné par une 1forme $\omega$ intégrable $(\omega \wedge d \omega=0)$ est un $\mathcal{D}$-groupoïde de Lie. Son idéal est engendré par les composantes de $\Gamma^{*} \omega \wedge \omega$ ou encore, en coordonnées dans lesquelles $\omega=\sum \omega_{i} d x_{i}$, par $\frac{\left(\Gamma^{*} \omega\right)_{i}}{\left(\Gamma^{*} \omega\right)_{j}}-\frac{\omega_{i}}{\omega_{j}}$. La troisième inclusion est vérifiée en dehors du lieu d'annulation de $\omega$.

Les autres exemples que l'on pourrait donner sont des généralisations de ceux-ci en considérant les groupoïdes d'invariance (ou d'isométries) de structures géométriques d'ordre supérieur à un : voir [Gr], [Dum].

Étant donné un sytème d'équations aux dérivées partielles d'ordre $k: \mathcal{I}_{k}$, l'idéal $p r_{q} \mathcal{I}_{k}$ des équations d'ordre $k+q$ obtenues par dérivations de $\mathcal{I}_{k}$ peut contenir des équations d'ordre $k$ n'appartenant pas à $\mathcal{I}_{k}$. Ceci nous interdit de considérer les jets d'ordre $k$ solutions de $\mathcal{I}_{k}$ comme des jets de solutions formelles. Les systèmes différentiels ayant de bonnes propriétés d'intégrabilité formelle $\left(p r_{q} \mathcal{I}_{k} \cap \mathcal{O}_{J_{k+s}^{*}}=p r_{s} \mathcal{I}_{k}\right)$ sont les systèmes involutifs. Les théorèmes d'involutivité générique de Cartan-Kuranishi et de B. Malgrange nous assurent que n'importe quel système différentiel est équivalent à un système involutif en dehors d'une hypersurface de conditions initiales.

Soit $\mathcal{I}_{k}$ un système d'équations d'ordre $k$ tel que si $E \in \mathcal{I}_{k} \cap \mathcal{O}_{J_{k-1}(\Delta)}$ alors $D_{i} E \in \mathcal{I}_{k}$. On note $S_{k}$ la variété analytique définie par $\mathcal{I}_{k}$ d'anneau $\mathcal{O}_{S_{k}}=\mathcal{O}_{J_{k}} / \mathcal{I}_{k}$ et pour $E$ dans $\mathcal{O}_{J_{k}}$ on note $\delta E$ le symbole de $E$, c'est-à-dire sa différentielle modulo les $d x_{i}$ et les $d y_{j}^{\alpha}$ pour $|\alpha| \leq k-1$. Soient $E_{1}, \ldots E_{p}$ un sytème d'équations qui engendre localement $\mathcal{I}_{k}$. Le premier prolongement de l'idéal est engendré par les $E_{\ell}$ et les $D_{i} E_{\ell}$. Pour trouver un zéro de $\operatorname{pr}_{1} \mathcal{I}_{k}$ dans $J_{k+1}^{*}(\Delta)$ au-dessus d'un zéro de $\mathcal{I}_{k}$ dans $J_{k}^{*}(\Delta)$, il faut résoudre un système d'équations

$$
\sum_{j,|\alpha|=k} \frac{\partial E}{\partial y_{j}^{\alpha}} y_{j}^{\alpha+\epsilon_{i}}=\sum_{j,|\alpha|<k} \frac{\partial E}{\partial y_{j}^{\alpha}} y_{j}^{\alpha+\epsilon_{i}} .
$$

De même, pour que $p r_{2} \mathcal{I}_{k}$ ait des zéros au-dessus de ceux de $p r_{1} \mathcal{I}_{k}$, il faut résoudre des équations de la forme

$$
\sum_{j,|\alpha|=k} \frac{\partial E}{\partial y_{j}^{\alpha}} y_{j}^{\alpha+\epsilon_{i}+\epsilon_{\ell}}=* .
$$

La nature des prolongements successifs de $\mathcal{I}_{k}$ est donc controlée par les symboles.

On note $A[\xi]$ l'anneau des polynômes en $\xi_{1}, \ldots \xi_{n}$ à coefficients dans un anneau $A$ et $A[\xi]_{k}$ l'espace des polynômes homogènes de degré $k$.

Définition 1.6. Après la substitution de $\delta y_{j}^{\alpha}$ par $\xi^{\alpha} \delta y_{j}$,

- on appelle symbole d'ordre $k$ de $\mathcal{I}_{k}$ le $\mathcal{O}_{S_{k}}$-module $N_{k}$ engendré dans

$\oplus_{j} \mathcal{O}_{S_{k}}[\xi]_{k} \delta y_{j}=\mathcal{O}_{S_{k}}[\xi]_{k}^{m}$ par les classes modulo $\mathcal{I}_{k}$ des $\delta f ;$

- on appelle symbole de $\mathcal{I}_{k}$ le $\mathcal{O}_{S_{k}}$-module gradué $N$ engendré par

$N_{k}$ dans $\mathcal{O}_{S_{k}}[\xi]^{m}$

- on appelle module caractéristique de $\mathcal{I}_{k}$, le module gradué quotient $M_{k}=\mathcal{O}_{S_{k}}[\xi]^{m} / N$. 
Dans la définition des systèmes différentiels ayant de bonnes propriétés de prolongement, les symboles interviendront par l'intermédiaire des évaluations ponctuelles du module caractéristique sur $S_{k}$. On demandera à ces derniers d'être involutifs.

Définition 1.7. Soit $M$ un $\mathbb{C}[\xi]$-module gradué. On dira que $M$ est $\ell$-involutif si il existe une base $\left(\eta_{1}, \ldots, \eta_{n}\right)$ de $\mathbb{C}[\xi]_{1}$ vérifiant pour tout $q \geq \ell$ :

Pour $i=1 \ldots n$, la multiplication par $\eta_{i}$

$$
M_{q} /\left(\eta_{1}, \ldots, \eta_{i-1}\right) M_{q-1} \rightarrow M_{q+1} /\left(\eta_{1}, \ldots, \eta_{i-1}\right) M_{q}
$$

est injective et $M_{q} /\left(\eta_{1}, \ldots, \eta_{n}\right) M_{q-1}=0$

Définition 1.8. On dira qu'un système différentiel $\mathcal{I}_{\ell}$ est $\ell$-involutif si :

(1) $S_{\ell}$ est lisse,

(2) $M_{\ell}$ et $M_{\ell+1}$ sont localement libres,

(3) en tout point a de $S_{\ell}, M(a)$ est $\ell$-involutif,

(4) $p r_{1} S_{\ell} \rightarrow S_{\ell}$ est surjectif.

Nous utiliserons les définitions précédentes uniquement à travers les trois théorèmes suivants. Pour leurs démonstrations, nous renvoyons le lecteur à [Ma6].

Théorème de Cartan-Kähler 1.9. Si $\mathcal{I}_{\ell}$ est $\ell$-involutif alors $\mathrm{pr}_{1} \mathcal{I}_{\ell}$ est $\ell+1$-involutif. Pour tout jet d'ordre $\ell$ solution de $\mathcal{I}_{\ell}$, il existe une solution convergente de $\mathcal{I}_{\ell}$ ayant ce jet d'ordre $\ell$.

Théorème d'involutivité générique 1.10 ([Ma6]). Soit $\mathcal{I}$ un idéal différentiel, réduit de $\mathcal{O}_{J^{*}(\Delta)}$ tel que les idéaux $\mathcal{I}_{k}$ soient cohérents. Quitte à diminuer le polydisque $\Delta$, il existe un entier $\ell$ et un sous-ensemble analytique fermé de codimension un $Z_{\ell} \subset S_{\ell}$ vérifiant :

(1) en dehors de $Z_{\ell}, \mathcal{I}$ est $\ell$-involutif,

(2) pour tout entier $q$, en dehors de $Z_{\ell}, \mathcal{I}_{\ell+q}$ est le prolongement d'ordre $q$ de $\mathcal{I}_{\ell}$ et il n'y a aucune composante de $\mathcal{I}_{\ell+q}$ au-dessus de $Z_{\ell}$.

B. Malgrange montre parallèlement une version analytique du théorème de RittRadenbush.

Théorème 1.11. Soit $\mathcal{I}$ un idéal différentiel réduit de $\mathcal{O}_{J^{*}(\Delta)}$. Quitte à réduire $\Delta$, il existe un entier $\ell$ tel que l'idéal $\mathcal{I}$ soit l'idéal réduit différentiablement engendré par $\mathcal{I}_{\ell}$.

Une première conséquence de ces théorèmes est le théorème suivant.

Théorème 1.12 ([Ma4]). Soit $\mathcal{I}_{k}$ un système différentiel d'ordre $k$. On note $\mathcal{I}$ l'idéal différentiel qu'il engendre et $\mathcal{I}^{\text {réd }}$ l'idéal réduit de $\mathcal{I}$. Supposons que $\mathcal{I}_{k}$ soit inclus dans ker $e^{*}$, soit stable par $i^{*}$ et qu'il existe un ensemble analytique fermé $Z$ de $\Delta$ tel que $\mathcal{I}_{k}$ définisse un groupoïde d'ordre $k$ en dehors de $Z$. Alors

(1) $\mathcal{I}^{\text {réd }}$ est l'idéal d'un $\mathcal{D}$-groupoïde de Lie,

(2) il existe un ensemble analytique fermé $Z^{\prime}$ de $\Delta$ tel que en dehors de $Z^{\prime}, \mathcal{I}=\mathcal{I}^{\text {réd. }}$.

Le théorème de "noethérianité" 1.11 permet de montrer :

Théorème 1.13 ([Ma4]). Soient $\mathcal{I}^{\alpha}$ des idéaux de $\mathcal{D}$-groupoïdes de Lie. L'idéal réduit engendré par la somme des $\mathcal{I}^{\alpha}$ est encore l'idéal d'un $\mathcal{D}$-groupoïde de Lie. 
Introduisons maintenant la notion de $\mathcal{D}$-algèbre de Lie, i.e la partie infinitésimal des $\mathcal{D}$-groupoïde de Lie. Commençons par définir le crochet de Spencer sur les section de l'espace des jets d'ordre $k$ de champs de vecteurs $J_{k}(\Delta \rightarrow T \Delta)$ en suivant la construction diagonale [K-S].

Soit $R_{k}$ le fibré sur $\Delta$ des jets d'ordre $k$ d'applications inversibles de $\Delta$ dans $\left(\mathbb{C}^{n}, 0\right)$. On a l'application suivante

$$
\lambda: R_{k} \times R_{k} \rightarrow J_{k}^{*}(\Delta)
$$

definie par $\left(\varphi_{2}, \varphi_{2}\right) \mapsto \varphi_{1} \circ \varphi_{2}^{-1}$. C'est le quotient de $R_{k} \times R_{k}$ sous l'action du groupe algébrique des jets d'ordre $k$ de biholomorphisme de $\left(\mathbb{C}^{n}, 0\right)$ :

$$
G L_{n}^{(k)}=J_{k}^{*}\left(\left(\mathbb{C}^{n}, 0\right) \rightarrow\left(\mathbb{C}^{n}, 0\right)\right)
$$

par composition aux buts sur les deux facteurs. Cette application induit une application du tangent vertical le long de la diagonale $T\left(R_{k} \times R_{k}\right) /\left.R_{k}\right|_{\text {diag }}$ sur le tangent vertical le long de l'identité $T\left(J_{k}^{*}(\Delta)\right) /\left.\Delta\right|_{i d}$ qui permet d'identifier les champs de vecteurs tangent à $J_{k}^{*}(\Delta)$ le long de l'identité et verticaux aux champs de vecteurs tangents à $R_{k} \times R_{k}$ le long de la diagonale, verticaux et invariant sous l'action de $G L_{n}^{(k)}$. On identifie ensuite le tangent vertical de $J_{k}^{*}(\Delta)$ le long de l'identité à l'espace des jets d'ordre $k$ de champs de vecteurs $J_{k}(\Delta \rightarrow T \Delta)$ de la manière suivante. À un jet d'ordre $k$ de champ vertical en $a, \sum b_{i}^{\alpha} \frac{\partial}{\partial y_{i}^{\alpha}}$, on fait correspondre le jet $\sum b_{i}^{\alpha}(x-a)^{\alpha} \frac{\partial}{\partial x_{i}}$.

Définition 1.14. Grâce aux construction ci-dessus, on peut identifier les sections de l'espace des jets $J_{k}(\Delta \rightarrow T \Delta)$ aux champs de vecteurs sur $R_{k}$ invariant sous l'action de $G L_{n}^{(k)}$. Ceci définit un crochet [, ] sur les sections de $J_{k}(\Delta \rightarrow T \Delta)$ en ramenant le crochet de Lie. C'est le crochet de Spencer. Ce crochet vérifie $\left[X^{(k)}, Y^{(k)}\right]=[X, Y]^{(k)}$ pour les couples de champs de vecteurs sur $\Delta$ où $(k)$ désigne la section donnée par le jet d'ordre $k$ d'un champ.

Définition 1.15. La $\mathcal{D}$-algèbre de Lie d'un $\mathcal{D}$-groupoïde de Lie d'idéal $\mathcal{I}$ est le linéarisé $d u \mathcal{D}$-groupoïde le long de l'identité. Elle est donnée par l'idéal linéaire et différentiel $\mathcal{L}(\mathcal{I})$ de $\mathcal{O}_{J^{*}(\Delta \rightarrow T \Delta)}$ engendré par les équations

$$
\mathcal{L}(E)=\sum_{i=1}^{n}\left(\frac{\partial E}{\partial y_{i}}(x, x, i d, 0, \ldots, 0) a_{i}+\sum_{|\alpha| \leq k} \frac{\partial E}{\partial y_{i}^{\alpha}}(x, x, i d, 0, \ldots, 0) a_{i}^{\alpha}\right)
$$

pour $E$ appartenant à $\mathcal{I}_{k}$. Les $a_{i}$ sont les coordonnées sur les fibres de $T \Delta$ induites par le choix de coordonnées $x_{i}$ sur $\Delta$.

Une solution de $\mathcal{L}(\mathcal{I})$ est un morphisme de $\mathcal{O}_{J^{*}(\Delta \rightarrow T \Delta)} / \mathcal{L}(\mathcal{I})$ dans $\mathcal{O}_{\Delta, p}^{n}$ commutant aux dérivations. Le choix de la coordonnée $x$ sur $\Delta$ identifie $\mathcal{O}_{\Delta, p}^{n}$ aux germes de champs de vecteurs en $p$. Soient $\left(a_{i}(x)\right)_{1 \leq i \leq n}$ les images des $\left(a_{i}\right)_{1 \leq i \leq n}$ sous ce morphisme : le champ $\sum a_{i}(x) \frac{\partial}{\partial x_{i}}$ est appelé champ solution de $\mathcal{L}(\mathcal{I})$.

Proposition 1.16 ([Ma4]). Soit $\mathcal{I}$ l'idéal d'un $\mathcal{D}$-groupoïde de Lie. Pour tout entier $\ell$, le crochet de Spencer de deux sections solutions de $\mathcal{L}(\mathcal{I})_{\ell}=\mathcal{L}(\mathcal{I}) \cap \mathcal{O}_{J_{\ell}^{*}(\Delta \rightarrow T \Delta)}$ est une section solution.

\section{Définitions 1.17.}

(1) Une $\mathcal{D}$-algèbre de Lie est donnée par un idéal linéaire différentiel $\mathcal{L}$ de $\mathcal{O}_{J^{*}}(\Delta \rightarrow T \Delta)$ tel que pour tout $\ell$, les sections du fibré vectoriel défini par le lieu d'annulation de $\mathcal{L}_{\ell}$ dans $J_{\ell}^{*}(\Delta \rightarrow T \Delta)$ soient stables par crochet de Spencer. 
(2) Nous dirons qu'une $\mathcal{D}$-algèbre de Lie est de rang $r$ lorsque le $\mathbb{C}$-espace vectoriel des champs solutions formelles en un point générique est de dimension $r$.

(3) Une $\mathcal{D}$-algèbre de Lie sera dite intégrable si elle est la $\mathcal{D}$-algèbre d'un $\mathcal{D}$-groupoïde de Lie.

(4) Un $\mathcal{D}$-groupoïde de Lie sera dit transitif lorsque les champs solutions de sa $\mathcal{D}$ algèbre de Lie évalués en un point générique $x$ engendrent $T_{x} \Delta$ (ou, de manière équivalente, lorsque son idéal ne contient pas d'équation d'ordre zéro).

La classification et l'étude des $\mathcal{D}$-groupoïdes de Lie au dessus d'un germe de disque de $\mathbb{C}$ est faite dans [Cas2] à partir des notes de B. Malgrange [Ma3]. Nous en rappelons les résultats principaux ci-dessous.

Proposition 1.18. Soit $\Delta$ un germe de disque de $\mathbb{C}$. Il y a exactement cinq types de $\mathcal{D}$-algèbres de Lie sur $\Delta$, correspondant aux équations suivantes :

$$
\begin{array}{lll}
\text { - rang } 0: a=0 & \text { notée } & A_{0} \\
\text { - rang } 1: a^{\prime}+\mu a=0 & \text { " } & A_{1}(\mu) \\
\text { - rang } 2: a^{\prime \prime}+\mu a^{\prime}+\mu^{\prime} a=0 & \text { " } & A_{2}(\mu) \\
\text { - rang } 3: a^{\prime \prime \prime}+\nu a^{\prime}+\frac{\nu^{\prime}}{2} a=0 & \text { " } & A_{3}(\nu) \\
\text { - rang } \infty: \text { équation nulle } & \text { " } & A_{\infty}
\end{array}
$$

les coefficents $\mu$ et $\nu$ étant méromorphes sur $\Delta$.

\section{Théorème 1.19 .}

(1) L'équation $\nu(y) y^{\prime 2}+2 \frac{y^{\prime \prime \prime}}{y^{\prime}}-3\left(\frac{y^{\prime \prime}}{y^{\prime}}\right)^{2}-\nu(x)=0$ définit l'unique $\mathcal{D}$-groupoïde de Lie ayant pour $\mathcal{D}$-algèbre de Lie $A_{3}(\nu)$ et sera noté $G_{3}(\nu)$. Dans ce cas, l'ensemble $Z$ (cf la définition 1.2) est le lieu des pôles de $\nu$.

(2) L'équation $\mu(y) y^{\prime}+\frac{y^{\prime \prime}}{y^{\prime}}-\mu(x)=0$ définit l'unique $\mathcal{D}$-groupoïde de Lie ayant pour $\mathcal{D}$-algèbre de Lie $A_{2}(\mu)$ et sera noté $G_{2}(\mu)$. Dans ce cas, $Z$ est le lieu des pôles de $\mu$.

(3) La D-algèbre de Lie $A_{1}(\mu)$ n'est intégrable que lorsque $\mu$ a un pôle simple de résidu rationnel $\frac{p}{q}$. Les $\mathcal{D}$-groupoïdes de Lie admettant $A_{1}(\mu)$ comme $\mathcal{D}$-algèbre de Lie sont alors définis par les équations :

$$
\gamma_{k}(y) y^{\prime k}-\gamma_{k}(x)=0 \text { avec } \gamma_{k}(x)=\exp \left(k \int \mu\right)(x)
$$

où $k$ est un entier multiple de $q$. Ils seront notés $G_{1}^{k}\left(\gamma_{k}\right)$. Dans ce cas, $Z$ est vide.

(4) Les $\mathcal{D}$-groupoïdes de Lie ayant une $\mathcal{D}$-algèbre de Lie de rang nul sont définis par une équation $h(x)-h(y)=0$ avec $h$ holomorphe sur $\Delta$. Ici $Z$ est encore vide.

Nous rappelons maintenant les résultats concernant les solutions $f:(\Delta, p) \rightarrow(\Delta, p)$ de ces $\mathcal{D}$-groupoïdes de Lie.

Proposition 1.20. Soit $a(x) \frac{d}{d x}$ un champ de vecteurs holomorphe sur un disque $\Delta$. Le germe de difféomorphisme $f=\exp \left(a(x) \frac{d}{d x}\right)$ est solution des $\mathcal{D}$-groupö̈des de Lie suivants :

$-\mathcal{G}_{1}\left(-\frac{1}{a}\right)$

- $\mathcal{G}_{2}(\mu)$ avec $\mu$ solution méromorphe de $a^{\prime \prime}+\mu a^{\prime}+\mu^{\prime} a=0$

- $\mathcal{G}_{3}(\nu)$ avec $\nu$ solution méromorphe de $a^{\prime \prime \prime}+\nu a^{\prime}+\frac{\nu^{\prime}}{2} a=0$ 
et seulement de ceux-ci.

Exemple 1.21. Les $\mathcal{D}$-groupoïdes de Lie d'idéaux non nuls contenant le difféomorphisme $\exp \left(x^{2} \frac{d}{d x}\right)$ sont $G_{1}\left(-\frac{2}{x}\right), G_{2}\left(-\frac{c}{x^{2}}-\frac{2}{x}\right)$ et $G_{3}\left(-\frac{c^{2}}{x^{4}}\right)$ ò̀ c est une constante d'intégration.

Le cas des germes de difféomorphismes formellement linéarisables est traité par l'énoncé suivant.

Théorème 1.22 ([Ma3]). Un difféomorphisme $f$ formellement linéarisable est solution d'un $\mathcal{D}$-groupoïde de rang fini si et seulement si $f$ est analytiquement linéarisable.

Dans ce cas $f$ est solution d'un groupoïde de rang $1: G_{1}\left(\frac{h^{\prime}}{h}\right)$ où $h$ est la linéarisante analytique de $f$.

Le cas d'un difféomorphisme $f$ parabolique, c'est-à-dire vérifiant $f^{\prime}(0)=1$, nécessite la mise en place des notations suivantes ([M-R2]). Nous noterons

$$
a_{k, \lambda}(x)=2 i \pi \frac{x^{k+1}}{1+\lambda x^{k}} \text { et } X_{k, \lambda}=a_{k, \lambda} \frac{d}{d x} .
$$

Les germes de difféomorphismes $g_{k, \lambda}=\exp \left(X_{k, \lambda}\right)$ sont les formes normales formelles des germes de difféomorphismes paraboliques. Ils admettent pour intégrales premières $H_{k, \lambda}(x)=x^{-\lambda} e^{1 / k x^{k}}$. Pour tout $f(z)=z+c z^{k+1}+\ldots$, il existe un difféomorphisme formel tangent à l'identité $\widehat{h}$ qui conjugue $f$ à $g_{k, \lambda}$. Un théorème d'Écalle, Voronin, Martinet-Ramis ([Ec],[Ma1]) dit que $\widehat{h}$ est k-sommable de somme $\left(U_{i}, h_{i}\right)$ sur $2 k$ secteurs $U_{i}$. On note $\operatorname{inv}(f)=\left(h_{i}\left(U_{i}\right) \cap h_{i+1}\left(U_{i+1}\right), h_{i+1} \circ h_{i}^{-1}\right)$ les invariants analytiques d'Écalle et Voronin définis à automorphisme près sur le disque du modèle formel et $s(f)=$ $\left(U_{i} \cap U_{i+1}, h_{i+1}^{-1} \circ h_{i}\right)$ leurs analogues sur $\Delta$.

\section{Proposition 1.23.}

(1) Si f est solution d'un $\mathcal{D}$-groupoïde de Lie, s(f) est solution du même $\mathcal{D}$-groupoïde de Lie.

(2) Soit $f$ de forme normale $g_{k, \lambda}$ et d'invariant analytique inv $(f)$. Le difféomorphisme $f$ est solution d'un $\mathcal{D}$-groupoïde de rang $r$ si et seulement si il existe un $\mathcal{D}$ groupö̈de de rang $r$ admettant $g_{k, \lambda}$ et inv $(f)$ comme solutions.

Le théorème suivant donne la liste des germes de difféomorphismes paraboliques solutions d'un $\mathcal{D}$-groupoïde de Lie de rang fini. Pour cela nous utiliserons les invariants "géométriques" $\left(\varphi_{i, i+1}\right)$ de Martinet-Ramis définis par $\varphi_{i, i+1} \circ\left(H_{k, \lambda} \circ h_{i+1}\right)=H_{k, \lambda} \circ h_{i}$. Ils sont alternativement définis au voisinage de 0 et de l'infini sur les sphères du chapelet décrivant l'espace des orbites de $f$ ([M-R2]).

\section{Théorème $\mathbf{1 . 2 4}$.}

(1) Un difféomorphisme tangent à l'identité est solution d'un $\mathcal{D}$-groupö̈de de Lie de rang 3 si et seulement si il existe un entier positif $p$ tel que ses invariants géométriques soient de la forme $\frac{\tau}{\sqrt[p]{1+a_{i} \tau^{p}}}$ en 0 et $\tau \sqrt[p]{1+\frac{b_{i}}{\tau^{p}}}$ en $\infty$. Suivant la terminologie de J. Écalle, de tels difféomorphismes seront appelés binaires.

(2) Un difféomorphisme tangent à l'identité est solution d'un $\mathcal{D}$-groupoïde de Lie de rang 2 si et seulement si il existe un entier $p$ tel que ses invariants géométriques soient tous de la forme $\frac{\tau}{\sqrt[p]{1+a_{i} \tau^{p}}}$ en 0 et $\tau$ en $\infty$ ou bien tous de la forme $\tau$ en 0 et $\tau \sqrt[p]{1+\frac{b_{i}}{\tau^{p}}}$ en $\infty$. De tels difféomorphismes seront appelés unitaires. 
Le cas des difféomorphismes résonnants, $f(x)=e^{2 i \pi \frac{p}{q}} x+\ldots$, se déduit de ce théorème en considérant $f^{\circ q}$.

\section{Le Groupoïde de Galois D’un feuilletage}

Soit $\mathcal{F}$ un germe de feuilletage holomorphe singulier de codimension $q$ défini par des formes $\omega_{1}, \ldots \omega_{q}$ indépendantes sur le corps des fonctions méromorphes vérifiant les conditions d'intégrabilité de Frobenius $d \omega_{i} \wedge \omega_{1} \wedge \ldots \wedge \omega_{q}=0$.

Le groupoïde d'holonomie d'un feuilletage est un objet transverse défini en dehors des singularités du feuilletage et il ne reflète pas complètement la complexité des singularités. Le groupoïde de Galois d'un feuilletage est la "clôture de Zariski" du groupoïde d'holonomie au sens de la définition suivante :

Définition 2.1 ([Ma4]). Soit $\mathcal{F}$ un germe de feuilletage holomorphe singulier de $\left(\mathbb{C}^{n}, 0\right)$. Son groupoïde de Galois, $\mathcal{G}$ al $(\mathcal{F})$, est le plus petit $\mathcal{D}$-groupoïde de Lie tel que les champs tangents à $\mathcal{F}$ soient solutions de sa $\mathcal{D}$-algèbre de Lie.

L'existence de cet objet est une conséquence direct du théorème 1.11. Quelques unes de ses propriétés ont été établies dans [Ma4].

Remarque 2.2. Les équations $\omega_{i}(X)=0$ définissant les champs tangents au feuilletage sont linéaires d'ordre zéro. L'hypothèse d'intégrabilité de Frobenius se traduit par le fait qu'elles définissent un faisceau en sous- $\mathbb{C}$-algèbre de Lie de $T \Delta$. L'idéal engendré par ces équations et toutes leurs dérivées définit une $\mathcal{D}$-algèbre de Lie, [Ma4]. Dans la plupart des cas cette $\mathcal{D}$-algèbre de Lie n'est pas intégrable.

Le $\mathcal{D}$-groupoïde de Lie $A u t\left(\mathcal{F}_{\omega}\right)$ des automorphismes du feuilletage a pour solutions les germes de difféomorphismes $\Gamma$ vérifiant $\Gamma^{*} \omega_{i} \wedge \omega_{1} \wedge \ldots \wedge \omega_{q}=0$. Il contient le groupoïde de Galois du feuilletage.

Définition 2.3. Un $\mathcal{D}$-groupoïde de Lie admissible pour $\mathcal{F}$ est un $\mathcal{D}$-groupoïde de Lie contenu dans $\operatorname{Aut}\left(\mathcal{F}_{\omega}\right)$ dont la $\mathcal{D}$-algèbre de Lie contient la $\mathcal{D}$-algèbre de Lie définie par le feuilletage.

Un tel $\mathcal{D}$-groupoïde de Liecontient donc le groupoïde de Galois du feuilletage. Nous allons étudier l'expression locale des équations d'un $\mathcal{D}$-groupoïde de Lie admissible sur un ouvert de redressement $U$. Nous noterons $t$ les coordonnées transverses et $z$ les coordonnées tangentes. Les $\mathcal{D}$-groupoïdes de Lie au-dessus de $U$ sont donnés par des équations sur les différents espaces de jets

$$
J_{k}^{*}(U)=U \times U \times G L_{n}(\mathbb{C}) \underset{2 \leq|\alpha| \leq k}{\times} \mathbb{C}^{n|\alpha|} .
$$

Nous noterons $(t, z)$ les coordonnées sur le premier $U,(T, Z)$ les mêmes coordonnées sur le second $U$ et $\frac{\partial^{|\alpha|+|\beta|}}{\partial t^{\alpha} \partial z^{\beta}} T, \frac{\partial^{|\alpha|+|\beta|}}{\partial t^{\alpha} \partial z^{\beta}} Z$ les coordonnées naturellement induites sur l'espace des jets.

\section{Lemme 2.4.}

(1) Soient $\mathcal{F}$ un feuilletage sur $\Delta$ et $(U,(t, z))$ un ouvert muni de coordonnées redressantes où $t$ désigne les coordonnées transverses et $z$ les coordonnées tangentes. L'idéal d'un $\mathcal{D}$-groupoïde de Lie admissible pour $\mathcal{F}$ est engendré sur $U$ par des équations

$$
\frac{\partial T_{j}}{\partial z_{i}}=0 \text { et } E_{i}\left(t, T, \ldots \frac{\partial^{|\alpha|} T}{\partial t^{\alpha}}\right)
$$


où les $E_{i}$ sont les équations d'un $\mathcal{D}$-groupoïde de Lie au-dessus du polydisque transverse $t(U)$.

(2) Le rang du $\mathcal{D}$-groupoïde transverse ainsi obtenu est indépendant de la carte choisie.

Définition 2.5. Ce rang est appelé rang transverse $d u \mathcal{D}$-groupoïde admissible.

Preuve. - Soit $\mathcal{I}$ l'idéal d'un $\mathcal{D}$-groupoïde de Lie admissible. Il s'obtient en complétant les équations de $A u t\left(\mathcal{F}_{\omega}\right): \frac{\partial T_{j}}{\partial z_{i}}=0$ par des équations supplémentaires $F_{i}(t, z, T, Z, \ldots)$. Toute solution $\Gamma$ de $\mathcal{I}$ se factorise sous la forme $\Gamma^{\text {trans }} \circ \Gamma^{\text {tang }}$ avec

$$
\Gamma^{\text {trans }}(t, z)=(T(t), z) \text { et } \Gamma^{\text {tang }}(t, z)=(t, Z(t, z)) \text {. }
$$

Comme $\mathcal{I}$ définit un $\mathcal{D}$-groupoïde admissible, toutes les transformations de la forme $\Gamma^{\text {tang }}$ sont solutions de $\mathcal{I}$. Pour toute solution $\Gamma$ de $\mathcal{I}, \Gamma^{\text {trans }}$ est aussi solution de $\mathcal{I}$ et vérifie de plus les équations de l'idéal différentiel engendré par $(Z-z)$. L'idéal différentiel $\mathcal{I}+(Z-z)$ décrit un $\mathcal{D}$-groupoïde de Lie et est engendré par des équations de la forme

$$
\frac{\partial T_{j}}{\partial z_{i}},\left(Z_{j}-z_{j}\right), E_{k}\left(z, t, T, \ldots, \frac{\partial^{|\alpha|} T}{\partial t^{|\alpha|}} \ldots\right) .
$$

Les translations $\tau(t, z)=(t, z+a)$ sont solutions de $\mathcal{I}$. La conjugaison par $\tau$ laisse $\mathcal{I}$ invariant ainsi que $(Z-z)$. L'idéal engendré par les équations ci-dessus est donc égal à l'idéal engendré différentiablement par

$$
\frac{\partial T_{j}}{\partial z_{i}},\left(Z_{j}-z_{j}\right), E_{k}\left(z_{0}, t, T, \ldots, \frac{\partial^{|\alpha|} T}{\partial t^{|\alpha|}} \ldots\right)
$$

Ceci permet de choisir des générateurs $E_{i}$ indépendants de $z$. Considérons l'idéal $\mathcal{J}=$ $\left(\frac{\partial T_{j}}{\partial z_{i}}, E_{k}\left(t, T, \ldots, \frac{\partial^{|\alpha|} T}{\partial t^{|\alpha|}}\right) \ldots\right)$. Comme $\mathcal{J}+(Z-z)=\mathcal{I}+(Z-z)$ est l'idéal d'un $\mathcal{D}$ groupoïde de Lie, l'idéal engendré au-dessus du disque tranverse par les équations $E_{i}\left(t, T, \ldots, \frac{d^{k} T}{d t^{k}} \ldots\right)$ définit un $\mathcal{D}$-groupoïde de Lie. Pour un entier $\ell$ assez grand, un jet d'ordre $\ell$ de transformation $\Gamma=\Gamma^{\text {trans }} \circ \Gamma^{\text {tang }}$ est solution de $\mathcal{J}_{\ell}$ si et seulement si $\Gamma^{\text {trans }}$ est solution de $\mathcal{I}_{\ell}$. La partie tangente $\Gamma^{\text {tang }}$ étant toujours solution de $\mathcal{I}_{\ell}, \mathcal{I}_{\ell}$ et $\mathcal{J}_{\ell}$ ont mêmes solutions parmi les jets d'ordre $\ell$. Comme ce sont des idéaux réduits, ils sont égaux. Les théorèmes 1.10 et 1.12 permettent de conclure que $\mathcal{I}=\mathcal{J}$.

Ce lemme ramène l'étude locale en un point régulier du feuilletage de son groupoïde de Galois à la compréhension des $\mathcal{D}$-groupoïdes de Lie définis au-dessus du polydisque transverse et de leurs prolongements analytiques sur le polydisque de définition du feuilletage. En particulier pour les feuilletages de codimension un, le groupoïde de Galois sera décrit par des $\mathcal{D}$-groupoïdes de Lie au-dessus d'un disque.

\section{Groupoïdes de Galois et Suites de Godbillon-Vey}

Dans ce paragraphe, $\mathcal{F}_{\omega}$ désigne le feuilletage holomorphe singulier donné par une 1forme $\omega$ intégrable $(\omega \wedge d \omega=0)$ sur un polydisque $\Delta$ dans $\mathbb{C}^{n}$. On pourra supposer que le lieu singulier de $\omega$ est de codimension deux.

Définition 3.1 ([G-V]). Une suite de Godbillon-Vey pour $\omega$ est une suite de 1-formes méromorphes $\omega_{1}, \omega_{2}, \ldots, \omega_{n}, \ldots$ telles que : 


$$
\begin{aligned}
d \omega & =\omega \wedge \omega_{1} \\
d \omega_{1} & =\omega \wedge \omega_{2} \\
& \vdots \\
d \omega_{n} & =\omega \wedge \omega_{n+1}+\sum_{k=1}^{n}\left(\begin{array}{c}
n \\
k
\end{array}\right) \omega_{k} \wedge \omega_{n-k+1}
\end{aligned}
$$

Elle sera dite de longueur $\ell$ si $\omega_{i}=0$ pour $i \geq \ell$, de longueur 1 si il existe $F$ méromorphe et un entier $k$ tels que $d\left(F^{1 / k} \omega\right)=0$.

On remarquera que les suites de longueur 1 sont des suites de longueur 2 particulières correspondant à $\omega_{1}=\frac{1}{k} \frac{d F}{F}$. La fonction multivaluée $F^{1 / k}$ est appelée facteur intégrant de la forme $\omega$. Sur $\Delta$ une telle suite existe toujours grâce à l'algorithme de Godbillon-Vey. Par contre l'existence d'une suite de longueur finie pour $\omega$ n'est pas toujours assurée. La longueur minimale des suites, mais pas la suite elle-même, ne dépend que du feuilletage $\mathcal{F}_{\omega}$. Nous ne nous intéresserons qu'aux suites de Godbillon-Vey de longueur inférieur ou égale à trois. Pour un étude des suites de longueur supérieure, on pourra consulter [CLLPT]. Les feuilletages de codimension un admettant une suite de Godbillon-Vey de longueur inférieure ou égale à trois sont caractérisés par leur groupoïde de Galois :

Théorème 3.2. Le feuilletage $\mathcal{F}_{\omega}$ admet une suite de Godbillon-Vey de longueur $\ell$ avec $\ell \leq 3$ si et seulement si son groupoïde de Galois est contenu dans un $\mathcal{D}$-groupoïde de Lie de rang transverse $\ell$.

Preuve du théorème 3.2 pour $\ell=1$. - Si $\mathcal{F}$ admet une suite de Godbillon-Vey de longueur 1, il existe $F$ méromorphe et un entier $k$ tels que $d\left(F^{1 / k} \omega\right)=0$. Soit $\Gamma$ un difféomorphisme local conservant le feuilletage, c'est-à-dire vérifiant $\Gamma^{*}(\omega)=f_{\Gamma} \omega$ pour une fonction $f_{\Gamma}$. On a alors $\Gamma^{*}\left(F^{1 / k} \omega\right)=F^{1 / k} \circ \Gamma f_{\Gamma} \omega$. Considérons l'équation d'invariance de la forme fermée :

$$
\Gamma^{*}\left(F^{1 / k} \omega\right)=F^{1 / k} \omega
$$

En prenant la puissance $k$-ième, nous obtenons l'équation à coefficients méromorphes :

$$
F=F \circ \Gamma f_{\Gamma}^{k}
$$

Ceci est l'équation d'un $\mathcal{D}$-groupoïde de Lie. En effet $f_{\Gamma}$ est un polynôme en les dérivées premières de $\Gamma$ à coefficients holomorphes en $\Gamma$ et méromorphes en $x$. De plus l'égalité

$$
f_{\Gamma_{1} \circ \Gamma_{2}}=\left(f_{\Gamma_{1}} \circ \Gamma_{2}\right) f_{\Gamma_{2}}
$$

permet de vérifier les axiomes d'un $\mathcal{D}$-groupoïde de Lie :

$$
F \circ\left(\Gamma_{1} \circ \Gamma_{2}\right) f_{\Gamma_{1} \circ \Gamma_{2}}^{k}-F \quad=\left(\left(F \circ \Gamma_{1} f_{\Gamma_{1}}^{k}-F\right) f_{\Gamma_{2}}^{k}\right) \circ \Gamma_{2}-\left(F \circ \Gamma_{2} f_{\Gamma_{2}}^{k}-F\right) .
$$

Vérifions que ce $\mathcal{D}$-groupoïde de Lie est admissible pour le feuilletage $\mathcal{F}_{\omega}$. Notons $L_{X}$ la dérivé de Lie par rapport à un champ $X$ et pour un champ $X$ préservant le feuilletage définissons $f_{X}$ par $L_{X} \omega=f_{X} \omega$. La $\mathcal{D}$-algèbre de Lie du groupoïde d'invariance de la forme fermée a pour équation :

$$
L_{X} F+k F f_{X}=0
$$


obtenue en linéarisant l'équation $F=F \circ \Gamma f_{\Gamma}^{k}$. Pour tout champ $X$ vérifiant $\omega(X)=0$, on a

$$
\begin{aligned}
d\left(F^{1 / k} \omega\right)(X, .) & =d\left(F^{1 / k}\right)(X) \omega+F^{1 / k} d \omega(X, .) \\
& =\left(L_{X} F^{1 / k}+F^{1 / k} f_{X}\right) \omega \\
& =\left(\frac{1}{k} \frac{L_{X} F}{F}+f_{X}\right) F^{1 / k} \omega .
\end{aligned}
$$

L'équation $d\left(F^{1 / k} \omega\right)=0$ est équivalente au fait que tout champ de vecteur tangent au feuillatage vérifie l'équation de la $\mathcal{D}$-algèbre de Lie. Par définition de $\mathcal{G} a l\left(\mathcal{F}_{\omega}\right)$, ce dernier est inclus dans le $\mathcal{D}$-groupoïde de Lie d'équations :

$$
\Gamma^{*} \omega \wedge \omega=0 \text { et } F^{k}=F^{k} \circ \Gamma f_{\Gamma}^{k} .
$$

Cette équation étant d'ordre un, son expression locale donne une équation d'ordre un qui correspond donc à un $\mathcal{D}$-groupoïde de Lie de rang transverse un.

Réciproquement, supposons que $\mathcal{F}$ admette un groupoïde de Galois de rang transverse un. Sur chaque carte de redressement de coordonnée transverse $t$, son équation, donnée par le (3) du théorème 1.19 et le lemme 2.4, est celle du $\mathcal{D}$-groupoïde de Lie d'invariance de la forme méromorphe $(\gamma(t) d t)^{\otimes k}$ où $\gamma$ est une racine $k$-ième de $\gamma_{k}$. Par transitivité, cet entier $k$ est indépendant de la carte locale. Les pôles et les zéros des différentes formes locales $\gamma^{\otimes k}$ se recollent en un ensemble analytique $Z$ de codimension un dans $\Delta-\operatorname{Sing}(\mathcal{F})$. Dans chaque carte de redressement du feuilletage ne rencontrant pas $Z$, définissons le facteur intégrant local par

$$
\Phi(z, t)=\frac{\gamma(t)}{w(z, t)}
$$

où $w$ est défini en coordonnées par $\omega=w(z, t) d t$.

Sur un autre ouvert muni de coordonnées redressantes $(\widetilde{z}, \widetilde{t})$, considérons la forme $\widetilde{\gamma}$ et le facteur intégrant $\widetilde{\Phi}$ définis de manière analogue.

Des changements de coordonnées :

$$
\begin{aligned}
\widetilde{\gamma}(\widetilde{t})\left(\frac{\partial \widetilde{t}}{\partial t}\right) & =\zeta \gamma(t) \text { avec } \zeta^{k}=1 \\
\text { et } \widetilde{w}(\widetilde{z}, \widetilde{t}) \frac{\partial \widetilde{t}}{\partial t} & =w(z, t),
\end{aligned}
$$

on déduit que les facteurs intégrants $\Phi$ se recollent en dehors de $Z$ à multiplication par une racine $k$-ième de l'unité près. La fonction $F=\Phi^{k}$ est bien définie et est méromorphe en dehors du lieu singulier du feuilletage. Celui-ci est de codimension deux et le théorème de Hartogs assure son prolongement méromorphe sur $\Delta$. Toute racine $k$-ième de cette fonction est un facteur intégrant et définit une suite de Godbillon-Vey de longueur un pour le feuilletage.

Remarque 3.3. Si $\mathcal{F}$ n'a pas d'intégrale première méromorphe, la forme $F^{1 / k} \omega$ est unique à multiplication par une constante près. Les équations du $\mathcal{D}$-groupö̈de que nous obtenons sont indépendantes de la forme $\omega$ initialement choisie. 
Preuve du théorème 3.2 pour $\ell=2$. $\quad$ Supposons que $\mathcal{F}$ admette une suite de Godbillon-Vey de longueur deux donnée par une forme $\alpha$ telle que $d \omega=\omega \wedge \alpha$ et $d \alpha=0$. Soit $(X, Y)=\Gamma(x, y)$ un germe de difféomorphisme local préservant le feuilletage, c'està-dire vérifiant :

$$
\Gamma^{*} \omega=f_{\Gamma} \omega
$$

Les égalités

$$
\begin{gathered}
\Gamma^{*} d \omega=\Gamma^{*} \omega \wedge \Gamma^{*} \alpha=-f_{\Gamma} \Gamma^{*} \alpha \wedge \omega \\
d \Gamma^{*} \omega=d f_{\Gamma} \wedge \omega+f_{\Gamma} d \omega=\left(d f_{\Gamma}-f_{\Gamma} \alpha\right) \wedge \omega
\end{gathered}
$$

donnent la relation

$$
\left(\Gamma^{*} \alpha-\alpha+\frac{d f_{\Gamma}}{f_{\Gamma}}\right) \wedge \omega=0
$$

entre les facteurs intégrants $\alpha$ pour $\omega$ et $\Gamma^{*} \alpha$ pour $\Gamma^{*} \omega$. Il existe donc une fonction $g_{\Gamma}$, déterminée par $\Gamma$, ses dérivées premières et secondes, vérifiant

$$
\Gamma^{*} \alpha-\alpha+\frac{d f_{\Gamma}}{f_{\Gamma}}=g_{\Gamma} \omega .
$$

On vérifie que les coefficients $g_{\Gamma}$ satisfont

$$
g_{\Gamma_{1} \circ \Gamma_{2}}=\left(g_{\Gamma_{1}} \circ \Gamma_{2}\right) f_{\Gamma_{2}}+g_{\Gamma_{2}} .
$$

Les transformations $\Gamma$ telles que $g_{\Gamma}=0$ sont donc solutions d'un $\mathcal{D}$-groupoïde de Lie d'ordre deux. Montrons que ce $\mathcal{D}$-groupoïde est admissible. Plus précisement, montrons qu'il contient $\mathcal{G} a l\left(\mathcal{F}_{\omega}\right)$ si et seulement si la forme $\alpha$ est fermée. En reprenant les notations du cas précédent, les équations linéarisées le long de l'identité de $g_{\Gamma}=0$ sont

$$
L_{X} \omega=f_{X} \omega \text { et } d f_{X}+L_{X} \alpha=0 .
$$

Prenons un champ $X$ tel que $\omega(X)=0$. On a alors :

$$
\begin{aligned}
L_{X} \omega & =d(\omega(X))+d \omega(X, .) \\
& =-\alpha(X) \omega \\
\text { donc } \quad d f_{X} & =-d(\alpha(X)) \\
\text { et } \quad L_{X} \alpha & =d(\alpha(X))+d \alpha(X, .) .
\end{aligned}
$$

Nous en déduisons que $X$ est solution du système linéarisé si et seulement si $d \alpha=0$. L'équation $g_{\Gamma}=0$ étant d'ordre deux, le $\mathcal{D}$-groupoïde de Lie admissible que nous venons de construire est de rang transverse deux.

Réciproquement, supposons qu'il existe un $\mathcal{D}$-groupoïde de Lie admissible de rang transverse deux. Nous allons utiliser l'expression locale de ce $\mathcal{D}$-groupoïde de Lie pour construire une intégrale première à monodromie affine. Plaçons nous sur un ouvert de redressement de coordonnées $(t, z)$ du feuilletage. La forme $\omega$ s'écrit $w(t, z) d t$ et les équations du $\mathcal{D}$-groupoïde de Lie admissible, données par lemme 2.4 et le théorème 1.19, sont de la forme :

$$
\frac{\partial T}{\partial z}=0 \text { et } \mu(t)=\mu(T) \frac{\partial T}{\partial t}+\frac{\frac{\partial^{2} T}{\partial t^{2}}}{\frac{\partial T}{\partial t}} .
$$

Quitte à le restreindre, supposons que cet ouvert soit simplement connexe et ne contienne pas de pôles de $\mu$. Considérons sur cet ouvert une intégrale première $H$ solution des 
équations :

$$
\frac{\partial H}{\partial z}=0 \text { et } \frac{\frac{\partial^{2} H}{\partial t^{2}}}{\frac{\partial H}{\partial t}}=\mu .
$$

Sur un ouvert analogue muni de coordonnées $(\widetilde{t}, \widetilde{z})$ nous construisons de même une intégrale première $\widetilde{H}$. Un calcul direct de changement de coordonnées dans les équations d'un $\mathcal{D}$-groupoïde de Lie sur un disque ([Cas2]) donne

$$
\widetilde{\mu}(\widetilde{t})=\mu(t) \frac{\partial t}{\partial \widetilde{t}}+\frac{\frac{\partial^{2} t}{\partial \tilde{t}^{2}}}{\frac{\partial t}{\partial \widetilde{t}}} .
$$

Ainsi $\widetilde{H}$ vérifie

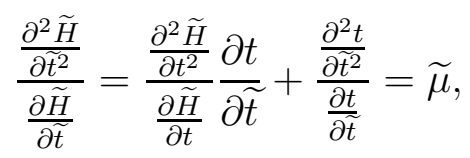

d'où

$$
\frac{\frac{\partial^{2} \widetilde{H}}{\partial t^{2}}}{\frac{\partial \widetilde{H}}{\partial t}}=\mu=\frac{\frac{\partial^{2} H}{\partial t^{2}}}{\frac{\partial H}{\partial t}} .
$$

Sur l'intersection des deux ouverts, que l'on suppose connexe, il existe donc deux constantes $a$ et $b$ telles que $\widetilde{H}=a H+b$. En prolongeant une solution locale par cette formule, on construit une intégrale première $H$ du feuilletage, en dehors du lieu des pôles des différents $\mu$, multivaluée à monodromie affine. La fonction $F=\frac{\frac{\partial H}{\partial t}}{w}$ vérifie

$$
d(F w d t)=0
$$

La forme fermée $\alpha=\frac{d F}{F}$ est univaluée en dehors des pôles des différents $\mu$ et vérifie $d \omega=\omega \wedge \alpha$. Sur une carte de redressement contenant des pôles de $\mu$, la forme $\alpha=\frac{d F}{F}$ se prolonge méromorphiquement. En effet,

$$
\alpha=\frac{d F}{F}=\frac{\frac{\partial^{2} H}{\partial t^{2}}}{\frac{\partial H}{\partial t}} d t-\frac{d w}{w}=\mu(t) d t-\frac{d w}{w},
$$

où les fonctions $\mu$ et $w$ sont méromorphes sur chaque carte de redressement. Le lieu singulier du feuilletage étant de codimension au moins deux, la forme $\alpha$ se prolonge méromorphiquement au polydisque.

Remarque 3.4. Les suites de Godbillon-Vey de longueur deux de la forme $(\omega, \alpha)$ et $\left(f \omega, \alpha-\frac{d f}{f}\right)$ sont équivalentes (voir $[\mathrm{Sc}]$ et $\left.[\mathrm{Go}]\right)$ : elles définissent la même structure affine transverse en dehors de $Z$. S'il n'existe pas de facteur intégrant méromorphe, la suite $(\omega, \alpha)$ est unique à équivalence près. L'équation du $\mathcal{D}$-groupoïde de Lie admissible de rang deux que nous obtenons est alors unique. 
Preuve du théorème 3.2 pour $\ell=3$. - Nous suivrons la même stratégie que dans les cas précédents. Supposons qu'il existe des formes méromorphes $\alpha$ et $\beta$ telles que :

$$
\begin{aligned}
& d \omega=\omega \wedge \alpha \\
& d \alpha=\omega \wedge \beta \\
& d \beta=\alpha \wedge \beta .
\end{aligned}
$$

Soit $\Gamma$ un automorphisme local du feuilletage et $f_{\Gamma}$ le coefficient de proportionnalité qu'il définit. De la première équation de Godbillon-Vey, nous déduisons l'existence d'une fonction $g_{\Gamma}$, déterminée par $\Gamma$, ses dérivées premières et secondes, vérifiant :

$$
\Gamma^{*} \alpha-\alpha+\frac{d f_{\Gamma}}{f_{\Gamma}}=g_{\Gamma} \omega .
$$

De la deuxième équation, nous déduisons les égalités :

$$
\begin{gathered}
d\left(\Gamma^{*} \alpha\right)=\omega \\
\wedge\left(f_{\Gamma} \Gamma^{*} \beta\right) \\
d\left(\alpha-\frac{d f_{\Gamma}}{f_{\Gamma}}+g_{\Gamma} \omega\right)=\omega \wedge \beta+d g_{\Gamma} \wedge \omega+g_{\Gamma} \omega \wedge \alpha .
\end{gathered}
$$

En faisant la différence, on obtient :

$$
\left(f_{\Gamma} \Gamma^{*} \beta-\beta+d g_{\Gamma}-g_{\Gamma} \alpha\right) \wedge \omega=0 .
$$

Il existe donc une fonction $h_{\Gamma}$ dépendant des dérivées troisièmes de $\Gamma$ telle que

$$
f_{\Gamma} \Gamma^{*} \beta-\beta-g_{\Gamma} \alpha+d g_{\Gamma}=h_{\Gamma} \omega .
$$

Nous en déduisons :

$$
h_{\Gamma_{1} \circ \Gamma_{2}}=h_{\Gamma_{2}}+h_{\Gamma_{1}} \circ \Gamma_{2}\left(f_{\Gamma_{2}}\right)^{2}+g_{\Gamma_{1}} \circ \Gamma_{2} g_{\Gamma_{2}} f_{\Gamma_{2}}
$$

d'où :

$$
h_{\Gamma_{1} \circ \Gamma_{2}}-\frac{1}{2}\left(g_{\Gamma_{1} \circ \Gamma_{2}}\right)^{2}=\left(h_{\Gamma_{2}}-\frac{1}{2}\left(g_{\Gamma_{2}}\right)^{2}\right)+\left(h_{\Gamma_{1}}-\frac{1}{2}\left(g_{\Gamma_{1}}\right)^{2}\right) \circ \Gamma_{2}\left(f_{\Gamma_{2}}\right)^{2} .
$$

L'équation $h_{\Gamma}-\frac{1}{2}\left(g_{\Gamma}\right)^{2}=0$ vérifie les axiomes d'un $\mathcal{D}$-groupoïde d'ordre trois. Montrons que ce $\mathcal{D}$-groupoïde contient $\mathcal{G} a l\left(\mathcal{F}_{\omega}\right)$ si et seulement si la troisième équation de la suite de Godbillon-Vey est vérifiée. La $\mathcal{D}$-algèbre de Lie de ce $\mathcal{D}$-groupoïde de Lie a pour équation :

$$
f_{X} \beta+L_{X} \beta-g_{X} \alpha+d g_{X}=0
$$

où $g_{X} \omega=d f_{X}+L_{X} \alpha$. Soit $X$ tel que $\omega(X)=0$. Comme dans le preuve précédente, on a $f_{X}=-\alpha(X)$. Nous déduisons

$$
g_{X} \omega=-d(\alpha(X))+d(\alpha(X))+d \alpha(X, .)=-\beta(X) \omega .
$$

Des égalités

$$
\begin{gathered}
d \beta(X, .)=L_{X} \beta+d\left(g_{X}\right) \\
\alpha \wedge \beta(X, .)=-f_{X} \beta+g_{X} \alpha
\end{gathered}
$$

on obtient par différence l'équation de la $\mathcal{D}$-algèbre de Lie sous la forme :

$$
(d \beta-\alpha \wedge \beta)(X, .)=0 .
$$

La troisième équation de la suite de Godbillon-Vey est donc équivalente au fait que tout champ tangent au feuilletage est solution de la $\mathcal{D}$-algèbre de Lie du $\mathcal{D}$-groupoïde de Lie que nous venons de construire. Ceci prouve l'inclusion de $\mathcal{G} a l\left(\mathcal{F}_{\omega}\right)$ dans un $\mathcal{D}$-groupoïde de Lie admissible de rang transverse trois. 
Réciproquement, supposons que le feuilletage admette un $\mathcal{D}$-groupoïde de Lie admissible de rang transverse trois. Sur un ouvert de redressement du feuilletage de coordonnées $(t, z)$, les équations du $\mathcal{D}$-groupoïde de Lie admissible, données par lemme 2.4 et le théorème 1.19, sont de la forme :

$$
\frac{\partial T}{\partial z}=0 \text { et } \nu(t)=\nu(T)\left(\frac{\partial T}{\partial t}\right)^{2}+S_{t}(T)
$$

où $S_{t}(T)=2 \frac{\frac{\partial^{3} T}{\partial t^{3}}}{\frac{\partial T}{\partial t}}-3\left(\frac{\frac{\partial^{2} T}{\partial t^{2}}}{\frac{\partial T}{\partial t}}\right)^{2}$ est la schwartzienne de $T$ par rapport à $t$. Nous allons nous servir de $\nu$ pour construire une intégrale première du feuilletage $H$ à monodromie projective et un couple de formes méromorphes $(\alpha, \beta)$ vérifiant les équations de Godbillon-Vey. Nous pouvons toujours choisir une forme méromorphe $\alpha$ vérifiant la première équation : il suffit de prendre un champ méromorphe $X$ vérifiant $\omega(X)=1$ et de poser $\alpha=L_{X} \omega$. Plaçons-nous sur un ouvert de redressement ne rencontrant pas le lieu des pôles $Z$ des différents $\nu$. Soit $H$ une intégrale première sur cet ouvert solution des équations :

$$
\frac{\partial H}{\partial z}=0 \text { et } S_{t} H=\nu(t)
$$

Soit $\widetilde{H}$ une intégrale première construite de manière analogue sur un ouvert de redressement muni des coordonnées $(\widetilde{t}, \widetilde{z})$. D'après les changements de variables usuels sur les dérivées Schwartziennes et sur les équations des $\mathcal{D}$-groupoïde de Lie [Cas2], nous avons

$$
S_{t} \widetilde{H}=S_{\widetilde{t}} \widetilde{H}\left(\frac{\partial t}{\partial \widetilde{t}}\right)^{2}+S_{\tilde{t}} t
$$

et

$$
\nu(t)=\widetilde{\nu}(\widetilde{t})\left(\frac{\partial t}{\partial \widetilde{t}}\right)^{2}+S_{\widetilde{t}} t
$$

Nous en déduisons que $S_{t} \widetilde{H}=S_{t} H$ et donc que $H$ se prolonge de manière multivaluée sur le complémentaire de $Z$ avec une monodromie projective. A partir de cette intégrale première, on construit la fonction $F$ par $d H=F \omega$ d'où :

$$
\frac{d F}{F} \wedge \omega+d \omega=0
$$

Contrairement au cas précédent, la forme $\frac{d F}{F}$ n'est pas méromorphe. La forme $\gamma=\frac{d F}{F}-\alpha$ vérifie $\gamma=G \omega$ pour une certaine fonction $G$. On a alors $d \alpha=\omega \wedge(d G-G \alpha)$. La forme $\beta$ cherchée est de la forme :

$$
\beta=d G-G \alpha+K \omega
$$

En remplaçant cette expression dans la troisième équation de Godbillon-Vey, on obtient :

$$
(d K+G d G) \wedge \omega+\left(G^{2}+2 K\right) \omega \wedge \alpha=0 .
$$

Posons $K=-\frac{1}{2} G^{2}$. Ceci nous permet de construire la forme $\beta$ à partir de $\alpha$ et $G$ vérifiant les équations de Godbillon-Vey. Il nous reste à montrer sa méromorphie. En prenant une autre détermination $\widetilde{H}=\frac{a H+b}{c H+e}$, nous obtenons, a priori, une autre forme $\widetilde{\beta}$. En calculant cette forme, on a : 


$$
\begin{aligned}
d \widetilde{H}=\widetilde{F} \omega & =\frac{F}{(c H+e)^{2}} \omega \\
\frac{d \widetilde{F}}{\widetilde{\widetilde{F}}} & =\frac{d F}{F}-2 \frac{c}{c H+e} d H \\
\widetilde{G} & =G-\frac{2 c F}{c H+e} \\
d \widetilde{G} & =d G-\frac{2 c d F}{c H+e}+\frac{2 c^{2} F^{2} \omega}{(c H+e)^{2}} \\
\widetilde{G} \alpha & =G \alpha-\frac{2 c F \alpha}{c H+e} \\
\frac{1}{2} \widetilde{G}^{2} \omega & =\frac{1}{2} G^{2} \omega-\frac{2 c G F \omega}{c H+e}+\frac{2 c^{2} F^{2} \omega}{(c H+e)^{2}} .
\end{aligned}
$$

En remplaçant $d F$ par $F \alpha+F G \omega$, on vérifie que $\beta=\widetilde{\beta}$. La forme $\beta$ est donc univaluée sur les ouverts de redressement ne rencontrant pas $Z$. Vérifions qu'elle admet un prolongement méromorphe sur $Z$. Sur une carte de redressement on écrit $\omega=w(t, z) d t$ et $\alpha=a_{t}(t, z) d t+a_{z}(t, z) d z$. Dans ces coordonnées $F=\frac{1}{w} \frac{\partial H}{\partial t}$ d'où :

$$
\begin{aligned}
& G w d t=\left(\frac{\partial_{t, t} H}{\partial_{t} H}-\frac{\partial_{t} w}{w}-a_{t}\right) d t+\left(\frac{\partial_{z} w}{w}+a_{z}\right) d z \\
& G= \frac{1}{w}\left(\frac{\partial_{t, t} H}{\partial_{t} H}-\frac{\partial_{t} w}{w}-a_{t}\right) \text { et } \frac{\partial_{z} w}{w}+a_{z}=0 \\
& d G=-\frac{\partial_{t} w}{w} G d t-\frac{\partial_{z} w}{w} G d z+\frac{1}{w}\left[\partial_{t}\left(\frac{\partial_{t, t} H}{\partial_{t} H}-\frac{\partial_{t} w}{w}-a_{t}\right)\right] d t \\
&+\frac{1}{w}\left(\partial_{z}\left(-\frac{\partial_{t} w}{w}-a_{t}\right)\right) d z \\
&-G \alpha=-a_{t} G d t-a_{z} G d z \\
&-\frac{1}{2} G^{2} \omega= \frac{1}{w}\left[-\frac{1}{2}\left(\frac{\partial_{t, t} H}{\partial_{t} H}\right)^{2}-\frac{1}{2}\left(\frac{\partial_{t} w}{w}\right)^{2}\right. \\
&\left.\quad-\frac{1}{2} a_{t}^{2}+\frac{\partial_{t, t} H}{\partial_{t} H} \frac{\partial_{t} w}{w}+\frac{\partial_{t, t} H}{\partial_{t} H} a_{t}-\frac{\partial_{t} w}{w} a_{t}\right] .
\end{aligned}
$$

En sommant les trois dernières équations, après simplification, on trouve :

$$
\beta=\frac{1}{w}\left[\nu(t) d t-d\left(\frac{\partial_{t} w}{w}+a_{t}\right)+\frac{1}{2}\left(\frac{\partial_{t} w}{w}+a_{t}\right)^{2} d t\right] .
$$

On en déduit que la forme $\beta$ est méromorphe en dehors du lieu singulier du feuilletage et se prolonge méromorphiquement à celui-ci.

Remarque 3.5. Les suites de Godbillon-Vey de longueur trois de la forme $(\omega, \alpha, \beta)$ et $\left(f \omega, \alpha-\frac{d f}{f}+g \omega, \frac{1}{f}\left(\beta-d g+g \alpha+\frac{g^{2}}{2} \omega\right)\right)$ sont équivalentes (voir [Sc] et [Go]) : elles définissent la même structure transverse projective en dehors de $Z$. Dans le cas où le feuilletage n'admet pas de suite de Godbillon-Vey de longueur deux, la suite de longueur trois est unique à équivalence près. Le $\mathcal{D}$-groupoïde de Lie obtenu est indépendant de la suite.

Remarque 3.6. Au cours de la preuve de ce théorème, nous avons donné les équations explicites d'un $\mathcal{D}$-groupoïde de Lie admissible pour le feuilletage ainsi que son expression locale sur une transverse. Soit $(t, z)$ des coordonnées de redressement. La forme $\omega$ s'écrit $w(t, z) d t$. L'expression locale du $\mathcal{D}$-groupö̈de de Lie transverse est donnée par les formules suivantes:

(1) Soit $\left(\omega, F^{1 / k}\right)$ une suite de longueur un pour le feuilletage. Le $\mathcal{D}$-groupoïde de Lie transverse est $\mathcal{G}_{1}(\mu)$ avec :

$$
\mu(t) d t=\frac{1}{k} \frac{d F}{F}+\frac{d w}{w} .
$$


(2) Soit $(\omega, \alpha)$ une suite de longueur deux pour le feuilletage. Le $\mathcal{D}$-groupoïde de Lie transverse est $\mathcal{G}_{2}(\mu)$ avec:

$$
\mu(t) d t=\alpha+\frac{d w}{w}
$$

(3) Soit $(\omega, \alpha, \beta)$ une suite de longueur trois pour le feuilletage. La forme $\alpha$ s'écrit $a_{t} d t+a_{z} d z$. Le $\mathcal{D}$-groupoïde de Lie transverse est $\mathcal{G}_{3}(\nu)$ avec :

$$
\nu(t) d t=w \beta+d\left(\frac{\partial_{t} w}{w}+a_{t}\right)-\frac{1}{2}\left(\frac{\partial_{t} w}{w}+a_{t}\right) d t .
$$

Réciproquement, ces mêmes formules permettent d'obtenir une suite de GodbillonVey explicite à partir des équations d'un $\mathcal{D}$-groupoïde de Lie admissible.

\section{GroupoÏDES DE Galois ET intÉGRALES PREMiÈRES}

Rappelons les types de transcendances d'extensions du corps des fonctions méromorphes sur un polydisque $\Delta$ de $\mathbb{C}^{n}$.

\section{Définitions 4.1.}

(1) Une extension différentielle du corps des fonctions méromorphes sera dite de type Darboux si elle est obtenue par une suite d'extensions qui sont soit algébriques soit du type $K(G) \supset K$ avec $d G=\gamma, \gamma$ étant une forme à coefficients dans $K$.

(2) Une extension différentielle du corps des fonctions méromorphes sera dite Liouvillienne si elle est obtenue par une suite d'extensions qui sont soit algébriques soit du type $K(G) \supset K$ avec $d G=G \gamma_{1}+\gamma_{0}, \gamma_{1}$ et $\gamma_{0}$ étant des formes à coefficients dans $K$.

(3) Une extension différentielle du corps des fonctions méromorphes sera dite de type Riccati si elle est obtenue par une suite d'extensions qui sont soit algébriques soit $d u$ type $K(G) \supset K$ avec $d G=\frac{G^{2}}{2} \gamma_{2}+G \gamma_{1}+\gamma_{0}, \gamma_{2}, \gamma_{1}$ et $\gamma_{0}$ étant des formes à coefficients dans $K$.

Théorème 4.2. Soit $\mathcal{F}$ un germe de feuilletage de codimension un de $\left(\mathbb{C}^{n}, 0\right)$. Le feuilletage $\mathcal{F}$ admet un intégrale première méromorphe (resp. de type Darboux, Liouville ou Riccati) si et seulement si $\mathcal{F}$ admet un $\mathcal{D}$-groupoïde de Lie admissible de rang transverse 0 (resp. 1,2 ou 3).

Nous allons commencer par prouver le cas non transitif (rang transverse 0). Les trois autres affirmations seront prouvées simultanément par la suite.

Lemme 4.3. Soient $\mathcal{F}$ un feuilletage de $\Delta$ de codimension un et $\mathcal{I}$ l'idéal du groupoïde de Galois de $\mathcal{F}$. Si le groupoïde de Galois n'est pas transitif alors l'idéal des équations d'ordre zéro, $\mathcal{I}_{0}=\mathcal{I} \cap \mathcal{O}_{J_{0}^{*}(\Delta)}$ de l'anneau $\mathcal{O}_{J_{0}^{*}(\Delta)}$, est engendré par une unique équation.

Preuve. - Plaçons-nous au voisinage d'un point régulier du feuilletage. D'après la forme des équations locales du $\mathcal{D}$-groupoïde de Lie (voir le lemme 2.4 ), et le fait que sa $\mathcal{D}$ algèbre de Lie soit de rang transverse nul, l'idéal $\mathcal{I}$ est engendré par une équation d'ordre 0 (voir le (4) du théorème 1.19). Ceci signifie que l'idéal $\mathcal{I}$ est engendré au voisinage de tout point de l'identité $\{(x, x, i d, 0 \ldots, 0) \mid x \notin \operatorname{Sing}(\mathcal{F})\}$ dans $J^{*}(\Delta)$ par une équation d'ordre zéro. Nous allons étendre cette propriété à tout jet dont la source et le but en dehors d'un ensemble de codimension un. 
En utilisant le théorème d'involutivité générique pour les $\mathcal{D}$-groupoïdes de Lie (théorème 1.10) et le théorème de Cartan-Kähler (1.9), il existe un sous-ensemble analytique $Z$ de $\Delta$ et un entier $\ell$ tels que par tout point $a=(s(a), t(a), \ldots)$ de $J_{\ell}^{*}(\Delta)$ solution de $\mathcal{I}_{\ell}$ de source et but hors de $Z$ passe une solution convergente $\varphi$ de $\mathcal{I}_{\ell}$. Quitte à supposer $\ell$ assez grand, $\varphi$ est solution de $\mathcal{I}$ : en effet d'après le théorème 1.11, il existe un entier $\ell$ tel que $\mathcal{I}_{\ell}$ engendre différentiablement $\mathcal{I}$.

Par composition à la source, cette solution donne un isomorphisme d'un voisinage de $(t(a), t(a), i d, 0, \ldots)$ sur un voisinage de $a$ dans l'espace des jets d'ordre $\ell$. Puisque $\varphi$ est solution de $\mathcal{I}$ et que les zéros de $\mathcal{I}_{\ell}$ sont stables par composition en dehors de $Z$, cet isomorphisme se restreint en un isomorphisme local des espaces définis par $\mathcal{I}_{\ell}$ aux voisinages de ces mêmes points. Nous en déduisons qu'au voisinage de n'importe quel point au-dessus de source et but en dehors de $Z, \mathcal{I}$ est engendré par une équation d'ordre 0 . Le lieu des zéros $V$ de $\mathcal{I}_{0}$ est de codimension un dans $(\Delta-Z) \times(\Delta-Z)$. De plus cet ensemble analytique $V$ n'a pas de composante irréductible incluse dans $(Z \times \Delta) \cup(\Delta \times Z)$. Dans le cas contraire il existerait une fonction $f$ holomorphe sur $V$ nulle sur le complémentaire de la composante irréductible et non nulle sur celle-ci. Puisque le produit de cette fonction par une équation de $Z$ est identiquement nul sur $V, f$ serait une élément de torsion de $\mathcal{O}_{J^{*}(\Delta)} / \mathcal{I}$ pour une des deux projections. L'idéal $\mathcal{I}$ étant différentiel et réduit, on vérifie que $f$ doit être identiquement nulle. Le lieu des zéros de l'idéal réduit $\mathcal{I}_{0}$ étant de codimension un, il est donc engendré par une équation $H([\mathrm{Ei}])$.

Une relation d'équivalence analytique sur $\Delta$ est la donnée d'un idéal $I$ de $\mathcal{O}_{\Delta \times \Delta}$ qui s'annule sur la diagonale, qui est stable par la symétrie par rapport à la diagonale et qui vérifie la relation de transitivité suivante :

$$
p r_{2,3}^{*} \mathcal{I} \subset p r_{1,2}^{*} \mathcal{I}+p r_{1,3}^{*} \mathcal{I}
$$

où les $p r_{i, j}$ désignent les trois projections naturelles de $\Delta \times \Delta \times \Delta$ sur $\Delta \times \Delta$.

Lemme 4.4. Sous les hypothèses du lemme 4.3, il existe un sous-ensemble analytique $Z$ de $\Delta$ tel que l'idéal $\mathcal{I}_{0}=(H(x, y))$ définisse une relation d'équivalence analytique sur $\Delta-Z$.

Preuve. - L'idéal $\mathcal{I}_{0}$ étant formé des équations d'ordre zéro de l'idéal $\mathcal{I}$ décrivant un $\mathcal{D}$-groupoïde de Lie, les propriétés de réflexivité et de symétrie sont vérifiées. La stabilité par composition nous donne l'inclusion de $p r_{2,3}^{*} \mathcal{I}_{0}$ dans l'idéal différentiablement engendré par $p r_{1,2}^{*} \mathcal{I}_{0}+p r_{1,3}^{*} \mathcal{I}_{0}$. Il nous faut vérifier qu'il est inclus dans l'idéal algébriquement engendré par $p r_{1,2}^{*} \mathcal{I}_{0}+p r_{1,3}^{*} \mathcal{I}_{0}$. Soit $Z$, l'ensemble analytique en dehors duquel on a la stabilité par composition du $\mathcal{D}$-groupoïde de Lie (voir (3) de la définition 1.2). Plaçonsnous sur $(\Delta-Z) \times(\Delta-Z) \times(\Delta-Z)$ et considérons les équations $p r_{1,2}^{*} H=H(x, y)$ et $p r_{1,3}^{*} H=H(x, z)$. Quitte à augmenter $Z$, les formes verticales pour la première projection :

$$
\sum \frac{\partial H}{\partial y_{i}}(x, y) d y_{i} \text { et } \sum \frac{\partial H}{\partial z_{i}}(x, z) d z_{i}
$$

ne s'annulent pas. On note $\mathcal{I}$ l'idéal différentiel engendré par $(H(x, y), H(x, z))$ dans $\mathcal{O}_{J(\Delta \rightarrow \Delta \times \Delta)}$ et $\mathcal{I}_{k}$ les équations de $\mathcal{I}$ d'ordre inférieur ou égal à $k$. Le fait que ces formes soient non nulles et non colinéaires nous permet d'utiliser une généralisation du théorème des fonctions implicites (voir [To]) : pour tout zéro $(x, y, z)$ de $(H(x, y), H(x, z))$ dans $(\Delta-Z) \times(\Delta-Z) \times(\Delta-Z)$, on peut trouver un zéro de $\mathcal{I}$ au-dessus de celui-ci pour la 
projection $J_{k}(\Delta \rightarrow \Delta \times \Delta) \rightarrow \Delta \times \Delta \times \Delta$. Ceci signifie que $\mathcal{I}_{k} \cap \mathcal{O}_{J_{0}(\Delta \rightarrow \Delta \times \Delta)}$ coïncide avec l'idéal algébriquement engendré par $H(x, y)$ et $H(x, z)$ sur $(\Delta-Z) \times(\Delta-Z) \times(\Delta-Z)$ pour tout entier $k$. Les équations d'ordre zéro appartenant à $\mathcal{I}$ sont donc exactement celles de cet idéal.

Pour $\ell$ assez grand, $\mathcal{I}_{\ell}$ est un sous-groupoïde de $J_{\ell}^{*}(\Delta-Z)$, donc $H(y, z)$ appartient à $\mathcal{I}_{\ell}$. D'après ce qui précède $H(y, z)$ appartient à l'idéal engendré algébriquement par $H(x, y)$ et $H(x, z)$. Nous avons donc une relation d'équivalence en dehors de $Z$.

Preuve du cas méromorphe du théorème 4.2. - On suppose que le groupoïde de Galois est d'ordre 0. Soit $H$ une équation de l'idéal $\mathcal{I}_{0}$ donnée par le lemme 4.3 et $Z$ le sous ensemble analytique en dehors duquel on a la stabilité du $\mathcal{D}$-groupoïde de Lie par composition. Quitte à agrandir $Z$, nous supposerons qu'il contient le lieu singulier du feuilletage. On note $R$ le lieu des zéros de $H$ dans $(\Delta \times \Delta)$ et $\left.R\right|_{\Delta-Z}$ sa restriction sur $(\Delta-Z) \times(\Delta-Z)$. Montrons que le quotient de $(\Delta-Z)$ par $\left.R\right|_{\Delta-Z}$ est une surface de Riemann.

Par transitivité, les classes d'équivalence $p r_{2}\left(\left.p r_{1}^{-1}(p) \cap R\right|_{\Delta-Z}\right)$ sont constantes le long des feuilles du feuilletage. Les projections $\left.R\right|_{\Delta-Z}$ sur $\Delta-Z$ étant sans torsion, elles sont plates au-dessus d'une transverse en $p$ au feuilletage. Par transitivité elles sont plates sur un ouvert contenant $p$. D'après $[\mathrm{Fr}]$ elles sont ouvertes et en particulier le saturé pour $\left.R\right|_{\Delta-Z}$ d'un ouvert est un ouvert.

Pour prouver la séparabilité du quotient, on prend deux points $p$ et $q$ non équivalents. Soit $T$ une transverse au feuilletage en $p$. Le point (4) du théorème 1.19 nous assure que les classes d'équivalence de $p$ et de $q$ intersectent $T$ en un nombre fini de points. On peut donc séparer ces deux ensembles par des ouverts dans $T$ saturés pour la relation d'équivalence restreinte à $T$. Les saturés de ces ouverts donnent deux ouverts dans le quotient qui ne s'intersectent pas. Le quotient par $\left.R\right|_{\Delta-Z}$ est un espace topologique séparé.

La construction d'un atlas holomorphe de cartes locales sur cet espace se fait de la manière suivante. Au voisinage $U$ d'un point $p$ de $\Delta-Z$, il existe une fonction holomorphe $h$ constante sur les classes d'équivalence. On prolonge $h$ sur le saturé $\bar{U}=p r_{2}\left(p r_{1}^{-1}(U) \cap\right.$ $\left.\left.R\right|_{\Delta-Z}\right)$ du voisinage par $p r_{2_{*}}\left(\left.p r_{1}^{*}(h)\right|_{\left.R\right|_{\Delta-Z}}\right)$. Ceci nous définit une carte sur l'ouvert $\bar{U} \mathrm{du}$ quotient. Soient $\left(h_{1}, \overline{U_{1}}\right)$ et $\left(h_{2}, \overline{U_{2}}\right)$ deux cartes d'intersection non vide. Les applications $\left.h_{1}\right|_{\overline{U_{1}} \cap \overline{U_{2}}}$ et $\left.h_{2}\right|_{\overline{U_{1}} \cap \overline{U_{2}}}$ ont les mêmes hypersurfaces de niveau. Il existe une application holomorphe $F$ telle que $\left.h_{1}\right|_{\overline{U_{1}} \cap \overline{U_{2}}}=\left.F \circ h_{2}\right|_{\overline{U_{1}} \cap \overline{U_{2}}}$. Celle-ci définit un changement de carte holomorphe pour la variété quotient.

On note alors $S$ la surface de Riemann obtenue et $\pi: \Delta-Z \rightarrow S$ le passage au quotient. Montrons que quitte à rajouter des points à $S, \pi$ se prolonge à $\Delta-\operatorname{Sing} \mathcal{F}$. Si une composante irréductible de $Z$ est transverse au feuilletage, $\pi$ étant constante sur les feuilles, elle se prolonge à cette composante. Sinon, considérons une transverse $T$ au feuilletage en un point $p$ de cette composante. D'après le (4) du théorème 1.19 , il existe une coordonnée source $x$ sur $T$ et but $X$ sur $T$ dans laquelle l'équation $H$ sur $T \times T$ s'écrive $X^{k}-x^{k}=0$. Par construction de $S$, au voisinage de $p$ le passage au quotient est donné par :

$$
\begin{aligned}
\pi: T-\{p\} & \rightarrow S \\
x & \mapsto x^{k} .
\end{aligned}
$$

Le quotient $\pi$ admet donc un prolongement holomorphe sur $Z$.

Notons encore $S$ l'image de ce prolongement. En ramenant une fonction méromorphe 
de $S$ sur $\Delta-\operatorname{Sing} \mathcal{F}$, on obtient une intégrale première méromorphe qui se prolonge à $\Delta$. Réciproquement si $\mathcal{F}$ admet une intégrale première méromorphe, le groupoïde d'invariance de celle-ci est un $\mathcal{D}$-groupoïde de Lie admissible pour $\mathcal{F}$ d'ordre zéro : voir l'exemple 1.3 .

Précisons maintenant la condition nécessaire et suffisante sur l'équation d'ordre zéro $H$ du groupoïde de Galois pour que le feuilletage admette une intégrale première holomorphe.

Proposition 4.5. Lorsque le groupoïde de Galois de $\mathcal{F}$ est non transitif d'équation $H$, le feuilletage admet une intégrale première holomorphe si et seulement si $H(0, y)$ est non identiquement nulle.

Preuve. - Si $H(0, y)$ est non identiquement nulle, nous pouvons supposer que $H$ est non identiquement nulle le long de l'axe des $y_{n}$ et appliquer le théorème de préparation de Weierstrass afin d'écrire :

$$
H(x, y)=y_{n}^{k}+a_{k-1}(x, \bar{y}) y_{n}^{k-1}+\ldots+a_{0}(x, \bar{y})
$$

où $\bar{y}=\left(y_{1}, \ldots, y_{n-1}\right)$. Fixons deux points $x$ et $y$ en dehors de $Z$ tels que $H(x, y)=0$. En utilisant la transitivité de la relation d'équivalence, $H(x, y)=0$ implique qu'au voisinage de tout $z \in \Delta-Z$ il existe une unité $u(z)$ telle que $H(x, z)=u(z) H(y, z)$. Grâce aux normalisations de Weierstrass des polynômes $H(x, z)$ et $H(y, z)$ on obtient $a_{0}(x, \bar{z})=$ $a_{0}(y, \bar{z})$ pour tout $\bar{z}$. En particulier on a $a_{0}(x, 0)=a_{0}(y, 0)$. Cette fonction est non constante. En effet par symétrie, il existe une unité $v$ telle que $H(x, y)=v(x, y) H(y, x)$ d'où

$$
a_{0}(x, 0)=H(x, 0)=v(x, 0) H(0, x)=v(x, 0)\left(x_{n}^{k}+\ldots\right) .
$$

Les feuilles de $\mathcal{F}$ étant incluses dans les classes d'équivalence de la relation d'équivalence donnée par $H$, la fonction holomorphe $a_{0}(x, 0)$ est une intégrale première du feuilletage. Réciproquement, si le feuilletage admet une intégrale première holomorphe non constante $h(x)$, l'équation $h(x)-h(y)$ definit un $\mathcal{D}$-groupoïde de Lie contenant le groupoïde de Galois du feuilletage. Les zéros de $H(x, y)$ sont donc inclus dans ceux de $h(x)-h(y)$. En particulier pour $x=0$ ceci montre que $H(0, y)$ est non identiquement nulle.

Remarque 4.6. Cette preuve s'adapte au cas méromorphe, suivant les indications de B. Malgrange, en utilisant le théorème de Weiertrass où on considère les variables $x$ comme paramètres.

Les preuves des autres cas du théorème 4.2 (rang transverse 1, 2 ou 3) se déduisent du théorème 3.2 et du théorème ci-dessous :

Théorème 4.7. Un germe de feuilletage holomorphe singulier $\mathcal{F}$ de codimension un admet une intégrale première de type Darboux (resp. Liouville ou Riccati) si et seulement s'il admet une suite de Godbillon-Vey de longueur un (resp. deux ou trois).

Preuve. - Le cas Liouvillien est dû à M. Singer : [Si]. Sa généralisation au cas Riccati est faite dans [Cas1]. Nous allons donner la preuve du cas Darboux.

Supposons qu'il existe une intégrale première $H$ de type Darboux pour la forme $\omega$. On note $K \subset K_{1} \ldots \subset K_{n}$ la suite des extensions du corps $K$, telle que $H$ soit dans $K_{n}$. On supposera que cette suite est de longueur minimale parmi toutes les suites d'extensions de type Darboux nécessaires à la construction d'une intégrale première. 
L'extension $K_{n-1} \subset K_{n}$ ne peut pas être algébrique. Dans le cas contraire, $H$ serait algébrique sur $K_{n-1}$. On note $P(X)=X^{p}+a_{p-1} X^{p-1} \ldots+a_{0}$ son polynôme minimal; $H$ n'étant pas constante, il en serait de même pour au moins un des $a_{i}$. On aurait

$$
0=d P(H) \wedge \omega=\sum_{i} H^{i} d a_{i} \wedge \omega
$$

d'où par minimalité de $P, d a_{i} \wedge \omega=0$. L'existence des intégrales premières $a_{i}$ dans $K_{n-1}$ contredit la minimalité de la suite d'extensions. La dernière extension est donc transcendante.

Soit $G$ telle que $K_{n}=K_{n-1}(G)$ où $d G=\gamma$ est une forme à coefficients dans $K_{n-1}$. Lorsqu'on écrit $d H=F \omega$ dans $K_{n}$, on peut supposer que le facteur intégrant $F$ est dans $K_{n-1}$. En effet, en écrivant $F=a_{k} G^{k}+\ldots$ par division suivant les puissances croissantes de $G$ et en calculant $d(F \omega)$, par transcendance de $G$ on obtient $d\left(a_{k} \omega\right)=0$. On peut donc considérer la suite de longueur minimale donnée par les $K_{i}$ pour $i$ inférieur à $n-1$ et $K_{n}=K_{n-1}(H)$ avec $d H=a_{k} \omega$. Il existe donc un facteur intégrant pour $\omega$ dans l'avantdernier corps de la suite d'extension donnant une intégrale première de type Darboux.

Si l'extension $K_{n-1}$ de $K_{n-2}$ est purement transcendante, le même raisonnement assure l'existence d'un facteur intégrant pour $\omega$ dans $K_{n-2}$, ce qui contredit la minimalité de la suite. L'extension $K_{n-1}$ de $K_{n-2}$ est donc algébrique.

Soit $F$ un facteur intégrant de $\omega$ dans $K_{n-1}$. Il est algébrique sur $K_{n-2}$. On note $F_{1}, \ldots, F_{p}$ ses quantités conjuguées. Comme $d\left(F_{i} \omega\right)=0$, on a

$$
\frac{d\left(F_{1} \ldots F_{p}\right)}{F_{1} \ldots F_{p}} \wedge \omega=p d \omega \text {. }
$$

Le produit $\widetilde{F}=F_{1} \ldots F_{p}$ est un élément non nul de $K_{n-2}$ dont une racine $p$-ième $\sqrt[p]{\widetilde{F}}$ est un facteur intégrant pour $\omega$. On construit donc une nouvelle suite de longueur minimale en conservant les $n-2$ premières extensions et en remplaçant $K_{n-1}$ par $K_{n-2}(\sqrt[p]{\widetilde{F}})$ et $K_{n}$ par $K_{n-2}(\sqrt[p]{\widetilde{F}}, H)$ avec $d H=\sqrt[p]{\widetilde{F}} \omega$. Montrons que cette suite est de longueur deux, c'est-à-dire $K_{n-2}=K_{0}$.

Si l'extension $K_{n-2}$ de $K_{n-3}$ est algébrique, le raisonnement précédent permet de construire une fonction $\widetilde{F}$ dans $K_{n-3}$ dont une racine est un facteur intégrant pour $\omega$. On pourrait alors construire une suite de longueur $n-1$ qui contredirait la minimalité de la suite.

Si l'extension $K_{n-2}=K_{n-3}(G)$ est transcendante avec $d G$ à coefficients dans $K_{n-3}$. On écrit $\widetilde{F}=a_{k} G^{k}\left(1+a_{1} G^{-1}+\ldots\right)$. En faisant la division suivant les puissances décroissantes, on a

$$
\frac{d \widetilde{F}}{\widetilde{F}} \wedge \omega=\frac{d a_{k}}{a_{k}} \wedge \omega+k \frac{d G \wedge \omega}{G}+\frac{d\left(a_{1} G^{-1}+\ldots\right)}{\left(1+a_{1} G^{-1} \ldots\right)} \wedge \omega .
$$

Le dernier terme de la somme contient des puissances de $G$ inférieures ou égales à -1 . Le deuxième terme est de degré -1 en $G$. Par transcendance de $G$ on en déduit que $\frac{d a_{k}}{a_{k}} \wedge \omega=p d \omega$. A partir du facteur intégrant $\sqrt[p]{a_{k}}$ on construit une suite d'extensions de longueur $n-1$ contenant une intégrale première. Ceci contredit à nouveau la minimalité de la suite.

On a obtenu une suite de longueur deux $K_{0} \subset K_{1} \subset K_{2}$ avec $K_{1}=K_{0}(\sqrt[p]{\widetilde{F}})$ et $K_{2}=K_{1}(H)$ où $d H=\sqrt[p]{\widetilde{F}} \omega:$ il existe un élément de $K$ dont une racine est un facteur intégrant pour $\omega$. 
Réciproquement, une suite de Godbillon-Vey de longueur un permet par ces formules de construire une intégrale première de type Darboux pour $\omega$.

Ceci achève la peuve du théorème.

\section{Le GroupoÏde de Galois D’un Germe de feuilletage De $\left(\mathbb{C}^{2}, 0\right)$ À SINGULARITÉ RÉDUITE}

Nous allons maintenant déterminer les feuilletages sur un bidisque à singularité réduite dont le groupoïde de Galois est de rang transverse fini en fonction des invariants de leurs classes analytiques.

Définition 5.1. Un feuilletage $\mathcal{F}$ de $\left(\mathbb{C}^{2}, 0\right)$ sera dit à singularité réduite si il existe une forme $\omega$ définissant $\mathcal{F}$ dont la partie linéaire s'écrit dans de bonnes coordonnées :

(1) $\lambda_{1} x d y+\lambda_{2} y d x,\left(\lambda_{1}, \lambda_{2}\right) \in \mathbb{C}^{*} \times \mathbb{C}^{*}, \frac{\lambda_{1}}{\lambda_{2}} \notin \mathbb{Q}<0$,

(2) $y d x$.

La terminologie employée renvoie au théorème de réduction de Seidenberg [Se] : ces singularités sont les plus simples que l'on obtient après éclatements.

Les feuilletages de type (1) sont appelés des selles. Ils admettent deux courbes analytiques invariantes lisses et transverses dont les holonomies ont pour parties linéaires $e^{-2 i \pi \lambda_{1} / \lambda_{2}}$ et $e^{-2 i \pi \lambda_{2} / \lambda_{1}}$. Ces feuilletages ont des comportements différents suivant les valeurs de $\frac{\lambda_{1}}{\lambda_{2}}$. Lorsque $\frac{\lambda_{1}}{\lambda_{2}}$ n'est pas réel ou réel négatif non rationnel, on sait, d'après Poincaré, que le feuilletage est analytiquement linéarisable. Lorsque $\frac{\lambda_{1}}{\lambda_{2}}$ est réel irrationnel, le feuilletage est formellement linéarisable [Il]. Lorsque $\frac{\lambda_{1}}{\lambda_{2}}$ est rationnel ces feuilletages s'appellent selles résonnantes. Ils ne sont plus linéarisables, mais admettent les formes normales formelles suivantes :

$$
p\left(1+(\lambda-1)\left(x^{p} y^{q}\right)^{k}\right) y d x+q\left(1+\lambda\left(x^{p} y^{q}\right)^{k}\right) x d y .
$$

Les axes de coordonnées sont des courbes invariantes pour ces feuilletages. L'holonomie de la feuille $x=0$ calculée sur la transverse $(y=1, x=t)$ est le difféomorphisme

$$
f=e^{-2 i \pi p / q} \exp \left(\frac{t^{q k+1}}{1-\lambda \frac{q}{p} t^{q k}} \frac{d}{d t}\right) .
$$

La classe de conjugaison analytique de cette holonomie est un invariant complet de la classe analytique du feuilletage. D'après les résultats de [M-R2], après préparation du feuilletage, il existe des normalisations analytiques $h_{i}$ sur des secteurs $U_{i}$ de la forme $-\frac{\pi}{2}-\varepsilon<\arg \left(\left(x^{p} y^{q}\right)^{k}\right)<\frac{\pi}{2}+\varepsilon$ qui sont asymptotes à la normalisante formelle tangente à l'identité. Le cocycle $\left(U_{i} \cap U_{i+1}, h_{i+1} \circ h_{i}^{-1}\right)_{i}$ induit un invariant complet de la classe analytique du feuilletage.

Les feuilletages de type (2) sont appelés des nœud-cols. Ils ont pour formes normales formelles

$$
x^{k+1} d y-y\left(1-\lambda x^{k}\right) d x
$$

d'holonomie $f=\exp \left(\frac{t^{k+1}}{1-\lambda t^{k}} \frac{d}{d t}\right)$ calculée sur la transverse $(y=1, x=t)$ à la séparatrice forte $x=0$. D'après les résultats de [M-R1], après préparation du feuilletage, il existe des normalisations analytiques $h_{i}$ sur des secteurs $U_{i}$ de la forme $-\frac{\pi}{2}-\varepsilon<\arg \left(x^{k}\right)<\frac{\pi}{2}+\varepsilon$ qui sont asymptotes à la normalisante formelle tangente à l'identité. Elles définissent un cocycle qui est un invariant complet de la classe analytique du feuilletage. 
Dans le cas des selles résonnantes et des nœud-cols, les invariants de la classe de conjugaison analytique de l'holonomie de la séparatrice forte sont donnés par les composantes transverses des invariants analytiques du feuilletage.

Proposition 5.2. Soit $f$ l'holonomie d'un feuilletage à singularité réduite $\mathcal{F}$. Si $f$ est solution d'un $\mathcal{D}$-groupoïde de Lie $\mathcal{G}$ sur un disque transverse $T$ alors il existe un $\mathcal{D}$ groupoïde de Lie admissible pour $\mathcal{F}$ dont l'équation transverse au voisinage de $T$ coïncide avec celle de $\mathcal{G}$.

Preuve. - Nous commençons par déterminer la liste des $\mathcal{D}$-groupoïdes de Lie de rang transverse supérieur ou égal à un admissible pour le nœud-col modèle $\omega=x^{2} d y-y d x$. Le facteur intégrant $F=\frac{1}{x^{2} y}$ détermine un $\mathcal{D}$-groupoïde de Lie de rang transverse un obtenu en écrivant l'invariance de la forme fermée $F \omega$ :

$$
\frac{d Y}{Y}-\frac{d X}{X^{2}}=\frac{d y}{y}-\frac{d x}{x^{2}}
$$

Considérons les cartes $y \notin \mathbb{R}_{\leq 0}$ et $y \notin \mathbb{R}_{\geq 0}$. En choisissant deux déterminations de $\log y$, on les munit des coordonnées redressantes $t=\frac{x}{x \log y+1}$ et $z=y$. Dans ces coordonnées, on vérifie que les équations de ce $\mathcal{D}$-groupoïde de Lie s'écrivent :

$$
\frac{\partial T}{\partial z}=0 \text { et } \frac{1}{T^{2}} \frac{\partial T}{\partial t}=\frac{1}{t^{2}}
$$

On retrouve la formule (1) de la remarque 3.6 qui donne sur la partie transverse le $\mathcal{D}$-groupoïde de Lie $\mathcal{G}_{1}\left(-\frac{2}{t}\right)$.

Les $\mathcal{D}$-groupoïdes de Lie de rang transverse deux admissibles pour $\omega$ sont obtenus d'après le théorème 3.2 à partir de toutes les formes fermées $\alpha$ vérifiant $d \omega=\omega \wedge \alpha$. Dans le cas du nœud-col, ces formes s'écrivent

$$
\alpha=\frac{d F}{F}+c F \omega
$$

où $c$ est un nombre complexe quelconque. Considérons les cartes $y \notin \mathbb{R}_{\leq 0}$ et $y \notin \mathbb{R}_{\geq 0}$. Dans les coordonnées précédentes, $\omega=w(z, t) d t$. D'après la formule (2) de la remarque 3.6, l'équation transverse du $\mathcal{D}$-groupoïde de Lie associé à la suite de Godbillon-Vey $(\omega, \alpha)$ est $\mathcal{G}_{2}(\mu)$ avec $\mu(t) d t=\alpha+\frac{d w}{w}$. On vérifie que les équations de ce $\mathcal{D}$-groupoïde de Lie s'écrivent:

$$
\frac{\partial T}{\partial z}=0 \text { et } \mathcal{G}_{2}\left(-\frac{c}{t^{2}}-\frac{2}{t}\right)
$$

Les $\mathcal{D}$-groupoïdes de Lie de rang transverse trois sont obtenus en prenant toutes les formes $\alpha$ vérifiant la première équation de Godbillon-Vey et en complétant la suite par l'unique forme $\beta$ satisfaisant les deux dernières équations de Godbillon-Vey. On obtient les suites $\left(\omega=x^{2} d y-y d x, \alpha=\frac{d F}{F}+c F \omega, \beta=F^{2} \omega\right)$. Dans les cartes de coordonnées $(t, z)$ précédentes, d'après la formule $(3)$ de la remarque 3.6, l'équation transverse du $\mathcal{D}$-groupoïde de Lie définie par la suite $(\omega, \alpha, \beta)$ est $\mathcal{G}_{3}(\nu)$ avec

$$
\nu(t) d t=w \beta+d\left(\frac{\partial_{t} w}{w}+a_{t}\right)-\frac{1}{2}\left(\frac{\partial_{t} w}{w}+a_{t}\right)^{2} d t
$$

où $\omega=w(z, t) d t$ et $\alpha=a_{t} d t+a_{z} d z$. On en déduit que les équations de ce $\mathcal{D}$-groupoïde de Lie s'écrivent :

$$
\frac{\partial T}{\partial z}=0 \text { et } \mathcal{G}_{3}\left(-\frac{c^{2}}{t^{4}}\right)
$$


D'autre part, d'après le début de la section 6 , les $\mathcal{D}$-groupoïdes de Lie sur le disque transverse contenant l'holonomie $\exp \left(t^{2} \frac{d}{d t}\right)$ du nœud-col sont $\mathcal{G}_{1}\left(-\frac{2}{t}\right), \mathcal{G}_{2}\left(-\frac{c}{t^{2}}-\frac{2}{t}\right)$ et $\mathcal{G}_{3}\left(-\frac{c^{2}}{t^{4}}\right)$ et seulement ceux-ci. Ces deux listes étant identiques, nous avons montré la proposition pour le nœud-col $x^{2} d y-y d x$. Les autres formes normales de nœud-cols, $x^{k+1} d y-y\left(1-\lambda x^{k}\right) d x$, se ramènent au cas précédent par $(x, y) \mapsto\left(\frac{x}{1-\lambda x \log x}, y\right)$ et la ramification $(x, y) \mapsto\left(x^{k}, y\right)$. Les formes normales de selles résonnantes se ramènent aux nœud-cols par l'éclatement $(x, y) \mapsto(x y, y)$ et la ramification $(x, y) \mapsto\left(x^{p}, y^{q}\right)$. Tous ces feuilletages admettant des facteurs intégrants, on peut aussi dériver directement les équations des $\mathcal{D}$-groupoïdes de Lie admissibles pour un de ces feuilletages en considérant toutes les suites de Godbillon-Vey que l'on peut associer à ce feuilletage, à équivalence près.

Considérons un nœud-col dans la classe formelle de $x^{k+1} d y-y\left(1-\lambda x^{k}\right) d x$ dont l'holonomie n'est plus analytiquement normalisable, et supposons que celle-ci soit solution d'un $\mathcal{D}$-groupoïde de Lie de rang trois $\mathcal{G}_{3}(\nu)$. Nous allons construire un $\mathcal{D}$-groupoïde de Lie admissible pour le feuilletage de rang transverse trois. On sait d'après le théorème 1.23, que $f$ est solution d'un $\mathcal{D}$-groupoïde de Lie de rang trois si et seulement si sa forme normale formelle et ses invariants analytiques sont eux-mêmes solutions d'un $\mathcal{D}$ groupoïde de Lie de rang trois $\mathcal{G}_{3}(\bar{\nu})$. Soit $\widehat{h}$ la conjugante formelle entre $f$ et sa forme normale. On a

$$
\nu=\bar{\nu} \circ \widehat{h}\left(\widehat{h}^{\prime}\right)^{2}+S \widehat{h}
$$

Nous avons prouvé ci-dessus que ce $\mathcal{D}$-groupoïde de Lie se prolonge en un $\mathcal{D}$-groupoïde de Lie $\mathcal{G}$ admissible pour le feuilletage modèle de rang transverse trois.

Maintenant nous allons construire un $\mathcal{D}$-groupoïde de Lie admissible pour le feuilletage initial à partir de $\mathcal{G}$. Quitte à faire une conjugaison analytique, on peut supposer que la séparatrice forte du feuilletage a pour équation $x=0$. Le nœud-col est alors conjugué au-dessus des secteurs $\left(-\frac{\pi}{2}-\epsilon \leq \arg \left(x^{2 k}\right) \leq \frac{\pi}{2}+\epsilon\right)$ à sa forme normale formelle par des normalisantes sectorielles $h_{i}$, avec $i$ variant dans $\mathbb{Z} / 2 k \mathbb{Z}$, asymptotes à la normalisante formelle. On considère alors les $\mathcal{D}$-groupoïdes de Lie $h_{i}^{*} \mathcal{G}$ au-dessus de chaque secteur. Une fois que l'on s'est fixé les deux premières formes $(\omega, \alpha)$ d'une suite de Godbillon-Vey de logueur trois pour le nœud-col, ce $\mathcal{D}$-groupoïde de Lie est la donnée d'une troisième forme $\beta_{i}$ satisfaisant les équations de Godbillon-Vey. Montrons que sur les intersections de deux de ces secteurs les deux formes $\beta_{i}$ et $\beta_{i+1}$ coïncident. Puisque, d'après [M-R1], les composantes transverses de $h_{i} \circ h_{i+1}^{-1}$ sont les composantes du cocycle des invariants de l'holonomie $f$, celui-ci étant solution de $\mathcal{G}_{3}(\bar{\nu})$, l'automorphisme du feuilletage modèle $h_{i} \circ h_{i+1}^{-1}$ est solution de $\mathcal{G}$. Soit $(t, z)$ des coordonnées redressantes au voisinage d'un disque $(z=0)$ transverse à la séparatrice forte $(t=0)$. En écrivant les équations des $\mathcal{D}$-groupoïdes de Lie correspondants aux triplets $\left(\omega, \alpha, \beta_{i}\right)$ et $\left(\omega, \alpha, \beta_{i+1}\right)$ (formules 3.6) on obtient pour chacune des équations sur les secteurs transverses correspondant, $\mathcal{G}_{3}\left(\nu_{i}\right)$ et $\mathcal{G}_{3}\left(\nu_{i+1}\right)$ avec

$$
\nu_{i}=\bar{\nu} \circ h_{i}\left(h_{i}^{\prime}\right)^{2}+S\left(h_{i}\right)
$$

où on désigne par $h_{i}$ la composante transverse de la normalisante sectorielle $h_{i}$. Comme la composante transverse de $h_{i} \circ h_{i+1}^{-1}$ est solution de $\mathcal{G}_{3}(\bar{\nu}) \nu_{i}=\nu_{i+1}$, les fonctions $\nu_{i}$ et $\nu_{i+1}$ étant asymptotes à $\nu$, elles sont égales à cette dernière. La forme $\beta$ est ainsi bien définie et méromorphe. 
Les selles résonnantes se traitent exactement de la même manière : seuls les secteurs changent de formes et sont donnés par $\left(-\frac{\pi}{2}-\epsilon \leq \arg \left(\left(x^{p} y^{q}\right)^{k}\right) \leq \frac{\pi}{2}+\epsilon\right)$.

La preuve dans le cas d'une holonomie unitaire est analogue.

Proposition 5.3. Soit $\mathcal{F}$ un germe de feuilletage à singularité réduite.

(1) $\mathcal{F}$ admet un $\mathcal{D}$-groupoïde de Lie admissible de rang transverse un si et seulement son holonomie est analytiquement normalisable.

(2) $\mathcal{F}$ admet un $\mathcal{D}$-groupö̈de de Lie admissible de rang transverse deux si et seulement si son holonomie est unitaire.

(3) $\mathcal{F}$ admet un $\mathcal{D}$-groupoïde de Lie admissible de rang transverse trois si et seulement si son holonomie est binaire.

Preuve. - Soit $\mathcal{F}$ un feuilletage réduit admettant un $\mathcal{D}$-groupoïde de Lie de rang transverse fini. Celui-ci définit un $\mathcal{D}$-groupoïde de Lie de même rang contenant l'holonomie par le lemme 2.4. Lorque l'holonomie est un difféomorphisme résonnant, le théorème 1.24 nous assure qu'elle est normalisable, unitaire ou binaire suivant la valeur du rang. Dans le cas des holonomies formellement linéarisables, le théorème 1.22 nous assure qu'elle est analytiquement linéarisable. Pour la réciproque, considérons d'abord les feuilletages analytiquement linéarisables ou normalisables. Il admettent toujours un facteur intégrant et donc un $\mathcal{D}$-groupoïde de Lie admissible de rang transverse un.

Considérons ensuite les selles résonnantes et les nœud-cols d'holonomie unitaire ou binaire. Le $\mathcal{D}$-groupoïde de Lie donné par le théorème 1.24 s'étend grâce à la proposition 5.2 précédente en un $\mathcal{D}$-groupoïde de Lie admissible pour le feuilletage de rang transverse deux ou trois.

Nous obtenons ainsi une nouvelle preuve de la caractérisation sur les invariants analytiques des feuilletages à singularité réduite admettant une structure transverse affine méromorphe ou une structure transverse projective méromorphe.

Ces résultats ont déjà été obtenus en utilisant d'autres techniques dans [B-T] pour le cas transversalement affine et [Tou2] dans le cas transversalement projectif.

\section{GroupoÏDEs De Galois ET EXTEnsions Fortement Normales}

Dans cette section $\mathcal{M}_{\Delta}$ désigne le corps des fonctions méromorphes sur le polydisque $\Delta$ de $\mathbb{C}^{n}$. Considérons l'espace $J_{k}^{*}(\Delta \rightarrow \mathbb{C})$ des jets d'ordre $k$ d'applications submersives de $\Delta$ dans $\mathbb{C}$ (la notation $*$ désigne ici la propriété de submersivité). Le choix de coordonnées $x$ sur $\Delta$ et $H$ sur $\mathbb{C}$ nous permet d'identifier cet espace à un ouvert de $\Delta \times \mathbb{C} \times|\alpha| \leq k, \mathbb{C}^{\alpha}$ avec les coordonnées $H^{\alpha}$ naturellement associées au choix de $x$ et de $H$. Ces espaces sont munis de l'anneau des équations aux dérivées partielles d'ordre inférieur à $k, \mathcal{O}_{J_{k}^{*}(\Delta \rightarrow \mathbb{C})}=\mathcal{O}_{\Delta}\left[H, \ldots, H^{\alpha} \ldots\right]$ et pour chaque dérivation partielle $\frac{\partial}{\partial x_{i}}$ d'une

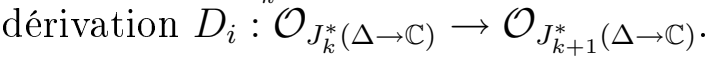

Définition 6.1. Une $\mathcal{D}$-variété dans $J^{*}(\Delta \rightarrow \mathbb{C})=\lim J_{k}^{*}(\Delta \rightarrow \mathbb{C})$ est donnée par un idéal $\mathcal{J} \subset \mathcal{O}_{J^{*}(\Delta \rightarrow \mathbb{C})}=\underset{\text { lim }}{\longrightarrow} \mathcal{O}_{J_{k}^{*}(\Delta \rightarrow \mathbb{C})}$ différentiel et réduit tel que $\mathcal{J} \cap \mathcal{O}_{\Delta}=\emptyset$.

Définition 6.2. Soit $\mathcal{A}$ un anneau différentiel sur $\mathcal{M}_{\Delta}$. Le spectre différentiel est l'ensemble $\operatorname{Spec}^{\operatorname{diff}}(\mathcal{A})$ des idéaux premiers et différentiels de $\mathcal{A}$. 
Cet ensemble peut être muni d'une topologie appelée topologie de Zariski-Kolchin ([Bu2], [Kov]). Nous allons étudier dans cette partie la $\mathcal{D}$-variété des intégrales premières d'un germe de feuilletage $\mathcal{F}$ défini par des formes $\omega_{1}, \ldots \omega_{q}$. Elle est donnée par le système d'équations aux dérivées partielles $d H_{i} \wedge \omega_{1} \wedge \ldots \wedge \omega_{q}=0$. Considérons l'anneau différentiel

$$
\mathcal{M}_{\Delta} \otimes_{\mathcal{O}_{\Delta}} \mathcal{O}_{J^{*}\left(\Delta \rightarrow \mathbb{C}^{q}\right)} /\left(d H_{i} \wedge \omega_{1} \wedge \ldots \wedge \omega_{q}\right)
$$

où $\left(d H_{i} \wedge \omega_{1} \wedge \ldots \wedge \omega_{q}\right)$ est l'idéal différentiel réduit engendré par les composantes de ces $(q+1)$-formes pour $i$ entre 1 et $q$, et notons $\mathcal{O}_{\mathcal{F}}$ son localisé sur $d H_{1} \wedge \ldots d H_{q} \neq 0$.

L'ensemble $\operatorname{Spec}{ }^{\operatorname{diff}} \mathcal{O}_{\mathcal{F}}$ représente l'ensemble des systèmes d'équations aux dérivées partielles compatibles avec le fait d'être un système complet d'intégrales premières. La notion de réductibilité d'un système d'équations aux dérivées partielles de Jules Drach ([Dr1]) correspond à la non trivialité du spectre différentiel.

Définition 6.3 ([Bu1],[Ko], [Kov]). Soit $\mathcal{M}_{\Delta} \subset \mathcal{K}$ une extension de degré de transcendance fini de corps différentiels. Cette extension sera dite fortement normale si pour toute extension différentielle $\mathcal{E}$ de $\mathcal{K}$ et tout morphisme $\sigma: \mathcal{K} \rightarrow \mathcal{E}$ au-dessus de $\mathcal{M}_{\Delta}$ :

(1) $\sigma$ laisse les constantes de $\mathcal{K}$ invariantes,

(2) $\sigma(\mathcal{K}) \cdot \mathcal{E}^{c}=\mathcal{K} \cdot \mathcal{E}^{c}$

où $\mathcal{E}^{c}$ désigne le corps des constantes de $\mathcal{E}$ et le point désigne le compositum des corps dans $\mathcal{E}$.

D'après la théorie de Kolchin (voir [Ko] et [Kov]), le groupe de Galois de ces extensions est un groupe algébrique. Ses sous-groupes algébriques sont en correspondance avec les extensions différentielles intermédiaires.

Le théorème suivant confirme les résutats incomplets de J. Drach [Dr2] et s'inscrit dans "une théorie générale de la réductibilité des équations" esquissée par E. Vessiot [Ves1], [Ves2].

Théorème 6.4. Soit $\mathcal{F}_{\omega}$ un feuilletage de codimension un de $\left(\mathbb{C}^{n}, 0\right)$. Les assertions suivantes sont équivalentes :

(1) le groupoïde de Galois de $\mathcal{F}_{\omega}$ est propre;

(2) le spectre différentiel de $\mathcal{O}_{\mathcal{F}}$ est non trivial : $\operatorname{Spec}^{\text {diff }}\left(\mathcal{O}_{\mathcal{F}}\right) \neq\{0\}$;

(3) il existe une intégrale première de $\mathcal{F}$ dans une extension fortement normale $\mathcal{K}$ de $\mathcal{M}_{\Delta}$.

Nous démontrerons successivement les implications $(3) \Rightarrow(2),(2) \Rightarrow(1)$ et $(1) \Rightarrow(3)$ dans les lemmes suivants. Nous montrerons les deux premières pour un feuilletage de codimension quelconque.

Lemme 6.5. Soit $\mathcal{F}$ un feuilletage donné par $q$ 1-formes. Si il existe $q$ intégrales premières fonctionnellement indépendantes dans une extension différentielle de $\mathcal{M}_{\Delta}$ de degré de transcendance fini alors le spectre différentiel de $\mathcal{O}_{\mathcal{F}}$ est non trivial.

Preuve. - L'existence d'un système d'intégrales premières dans $\mathcal{K}$ donne un morphisme différentiel au-dessus de $\mathcal{M}_{\Delta}$ :

$$
\mathcal{O}_{\mathcal{F}} \longrightarrow \mathcal{K}
$$

induit par l'identification des coordonnées $H_{i}$ avec les intégrales premières. Le noyau de ce morphisme est un idéal différentiel premier de $\mathcal{O}_{\mathcal{F}}$ et donne donc un élément de 
$\operatorname{Spec}^{\operatorname{diff}}\left(\mathcal{O}_{\mathcal{F}}\right)$. L'extension $\mathcal{K}$ étant de degré de transcendance fini ce qui n'est pas le cas de $\mathcal{O}_{\mathcal{F}}$, le morphisme ne peut pas être injectif. L'élément obtenu dans $\operatorname{Spec}^{\operatorname{diff}}\left(\mathcal{O}_{\mathcal{F}}\right)$ est non trivial.

Lemme 6.6. Soit $\mathcal{F}$ un feuilletage donné par q 1-formes. Si le spectre différentiel de $\mathcal{O}_{\mathcal{F}}$ est non trivial alors le groupoïde de Galois de $\mathcal{F}_{\omega}$ est propre.

Preuve. - Soit $\mathcal{J}$ un idéal différentiel premier de $\mathcal{O}_{J^{*}\left(\Delta \rightarrow \mathbb{C}^{q}\right)}$ contenant l'idéal différentiel donné par $d H_{i} \wedge \omega_{1} \wedge \ldots \omega_{q}$. Nous allons construire un $\mathcal{D}$-groupoïde de Lie dont les solutions sont les germes $\varphi$ tels que pour tout $H=\left(H_{1}, \ldots, H_{q}\right), H$ est solution de $\mathcal{J}$ si et seulement si $H \circ \varphi$ est solution de $\mathcal{J}$. Pour cela on considère l'action de $J_{p}^{*}(\Delta)$ sur $J_{p}^{*}\left(\Delta \rightarrow \mathbb{C}^{q}\right)$ par composition à la source :

$$
\text { comp : } J^{*}\left(\Delta \rightarrow \mathbb{C}^{q}\right) \times_{\Delta} J^{*}(\Delta) \longrightarrow J^{*}\left(\Delta \rightarrow \mathbb{C}^{q}\right) .
$$

Cette action se traduit sur les anneaux par l'existence d'une flèche comp* satisfaisant les diagrammes commutatifs suivant :

- l'identité

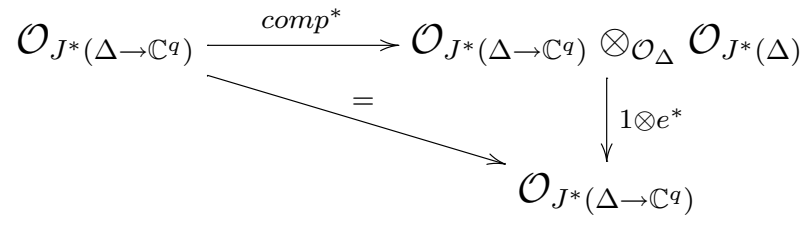

- la composition

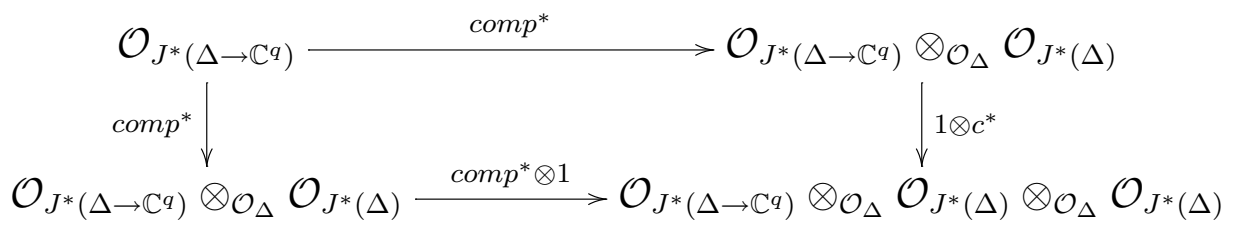

Un germe de difféomorphisme $\varphi:(\Delta, a) \rightarrow(\Delta, b)$ induit par prolongement un morphisme $\varphi^{*}$ des jets de $J^{*}\left(\Delta \rightarrow \mathbb{C}^{q}\right)$ de source $b$ sur ceux de source $a$ par composition. Étant donné un idéal $\mathcal{J}$ de $\mathcal{O}_{J_{p}^{*}\left(\Delta \rightarrow \mathbb{C}^{q}\right)}$, nous allons chercher à déterminer les équations différentielles satisfaites par les germes $\varphi$ tels que $\varphi^{* *}\left(\mathcal{J} \otimes \mathbb{C}_{a}\right)=\left(\mathcal{J} \otimes \mathbb{C}_{b}\right.$ ) (on note $\varphi^{* *}$ le morphisme d'anneau induit par la transformation $\left.\varphi^{*}\right)$. Considérons l'idéal comp $p^{*} \mathcal{J}$ dont les solutions sont l'ensemble des couples $(H, \varphi)$ tels que $H \circ \varphi$ est solution de $\mathcal{J}$. Les solutions de comp $^{*} \mathcal{J}+\mathcal{J} \otimes 1$ sont les couples $(H, \varphi)$ tels que $H$ et $H \circ \varphi$ soient solutions de $\mathcal{J}$. Il faut déterminer le plus petit idéal $\mathcal{I}$ de $\mathcal{O}_{J^{*}(\Delta)}$ vérifiant comp ${ }^{*} \mathcal{J}+\mathcal{J} \otimes 1 \subset \mathcal{J} \otimes 1+1 \otimes \mathcal{I}$ en dehors d'une hypersurface. Les solutions de $\mathcal{I}$ sont les $\varphi$ tels que si $H$ est solution de $\mathcal{J}$ alors $H \circ \varphi$ est solution de $\mathcal{J}$.

Nous allons déterminer un système de générateurs de l'idéal $\mathcal{I}$. En l'absence de torsion, nous noterons encore $\mathcal{J}$ l'idéal engendré par $\mathcal{J}$ dans $\mathcal{M}_{\Delta} \otimes \mathcal{O}_{J^{*}\left(\Delta \rightarrow \mathbb{C}^{q}\right)}$. D'après le théorème de "noetherianité" 1.11, il existe un entier $k$ tel que l'idéal $\mathcal{J}$ soit différentiablement engendré, en tant qu'idéal réduit, par ses éléments d'ordre inférieur à $p$. On note $\mathcal{J}_{p}$ la trace de l'idéal $\mathcal{J}$ dans l'anneau des équations différentielles d'ordre inférieur ou égale à $p: \mathcal{O}_{J_{p}^{*}\left(\Delta \rightarrow \mathbb{C}^{q}\right)}$.

Soit $f_{1}, \ldots, f_{n}$ un système générateur de l'idéal $\mathcal{J}_{p}$, qu'on supposera $\mathcal{M}_{\Delta}$-libre. On le complète en une base du $\mathcal{M}_{\Delta}$-espace vectoriel $\mathcal{J}_{p}: f_{1}, \ldots, f_{n}, \ldots, f_{m}, \ldots$ Puis on complète cette famille par des $e_{1}, \ldots, e_{k}, \ldots$ en une $\mathcal{M}_{\Delta}$-base de $\mathcal{M}_{\Delta} \otimes \mathcal{O}_{J_{p}^{*}\left(\Delta \rightarrow \mathbb{C}^{q}\right)}$. Soit $f \in \mathcal{M}_{\Delta} \otimes$ 
$\mathcal{O}_{J_{p}^{*}\left(\Delta \rightarrow \mathbb{C}^{q}\right)}$, nous noterons :

$$
c o m p^{*} f=\sum f_{j} \alpha^{j}(f)+\sum e_{k} \beta^{k}(f),
$$

avec les $\alpha^{j}(f)$ et $\beta^{k}(f)$ dans $\mathcal{M}_{\Delta} \otimes_{\mathcal{O}_{\Delta}} \mathcal{O}_{J_{p}^{*}(\Delta)}$. On considère alors l'idéal $\mathcal{I}_{p}$ de $\mathcal{M}_{\Delta} \otimes_{\mathcal{O}_{\Delta}}$ $\mathcal{O}_{J_{k}^{*}(\Delta)}$ défini par les $\beta^{k}(f)$ pour $f \in \mathcal{J}_{p}$. Par construction cet idéal vérifie deux propriétés importantes.

(a) Il est engendré par les $\beta^{k}\left(f_{i}\right)$ pour $i=1, \ldots, n$. En effet, on a clairement $\beta^{k}(f+$ $g)=\beta^{k}(f)+\beta^{k}(g)$. D'autre part comme

$$
\operatorname{comp}^{*}(f g) \equiv \sum e_{\ell} e_{k} \beta^{\ell}(f) \beta^{k}(g) \bmod \mathcal{J}_{p} \otimes 1
$$

en écrivant $e_{\ell} e_{k}$ dans la base décrite au-dessus, on obtient que $\beta^{i}(f g)$ est une combinaison des $\beta^{\ell}(f) \beta^{k}(g)$ à coefficients dans $\mathcal{M}_{\Delta}$. En particulier, quelque soit $f$ dans $\mathcal{J}_{p}, \beta^{k}(f)$ est dans l'idéal engendré par les $\beta^{k}\left(f_{i}\right)$ pour $i=1, \ldots, n$.

(b) En considérant la décomposition de $\operatorname{comp}^{*} f_{i}$ on remarque que, pour tout $\varphi$ de $J_{p}^{*}(\Delta)$ solution de $\mathcal{I}_{p}$, si $H$ est solution de $\mathcal{J}_{p}$ alors $H \circ \varphi$ est aussi solution de $\mathcal{J}_{p}$. Réciproquement si pour toute solution $H$ de $\mathcal{J}_{p}, H \circ \varphi$ est encore solution de $\mathcal{J}_{p}$ alors, en utilisant l'indépendance des $e_{k}$, on a $\varphi$ solution de $\mathcal{I}_{p}$. Un jet $\varphi$ est solution de $\mathcal{I}_{p}$ si et seulement si $\varphi^{* *} \mathcal{J}_{p} \otimes \mathbb{C}_{a} \subset \mathcal{J}_{p} \otimes \mathbb{C}_{b}$.

Vérifions maintenant que cet idéal vérifie les propriétés (1) et (3) d'un $\mathcal{D}$-groupoïde de Lie. L'inclusion de cet idéal dans l'idéal définissant l'identité se déduit du diagramme commutatif de l'identité ci-dessus. D'autre part on a :

$$
\begin{aligned}
\left(\mathrm{comp}^{*} \otimes 1\right)\left(\mathrm{comp}^{*}\right)(f)=\sum_{i, j} f_{i} \alpha^{i}\left(f_{j}\right) \alpha^{j}(f) & \sum_{l, j} e_{l} \beta^{l}\left(f_{j}\right) \alpha^{j}(f) \\
& +\sum_{i, k} f_{i} \alpha^{i}\left(e_{k}\right) \beta^{k}(f)+\sum_{k, l} e_{l} \beta^{l}\left(e_{k}\right) \beta^{k}(f)
\end{aligned}
$$

et

$$
\left(1 \otimes c^{*}\right)\left(c o m p^{*}\right)(f)=\sum f_{i} c^{*}\left(\alpha^{i}(f)\right)+\sum e_{l} c^{*}\left(\beta^{l}(f)\right) .
$$

En utilisant le second diagramme commutatif, on obtient les égalités

$$
c^{*}\left(\beta^{l}\left(f_{i}\right)\right)=\sum_{k} \beta^{l}\left(e_{k}\right) \beta^{k}\left(f_{i}\right)+\sum_{j} \beta^{l}\left(f_{j}\right) \alpha^{j}\left(f_{i}\right)
$$

qui prouvent la stabilité par composition. N'ayant pas de stabilité par l'inversion $i$, considérons l'idéal $\mathcal{I}_{p}+i^{*} \mathcal{I}_{p}$. Par construction il est stable par inversion et reste contenu dans l'idéal de l'identité. Nous venons de prouver qu'une partie de cet idéal est stable par composition. Pour prouver que l'autre partie l'est aussi on introduit l'application $i \widehat{\times} i$ définie de $J^{*}(\Delta) \times_{\Delta} J^{*}(\Delta)$ dans $J^{*}(\Delta) \times_{\Delta} J^{*}(\Delta)$ par :

$$
((x, y, \ldots),(y, z, \ldots)) \rightarrow((z, y, \ldots),(y, x, \ldots)) .
$$

Cette flèche induit un morphisme $(i \widehat{\times} i)^{*}$ vérifiant $(i \widehat{\times} i)^{*} c^{*}=c^{*} i^{*}$. En l'appliquant à l'égalité donnant la stabilité par composition de $\mathcal{I}_{p}$, on a :

$$
c^{*}\left(i^{*}\left(\beta^{l}\left(f_{i}\right)\right)\right)=\sum_{k} i^{*}\left(\beta^{k}\left(f_{i}\right)\right) i^{*}\left(\beta^{l}\left(e_{k}\right)\right)+\sum_{j} i^{*}\left(\alpha^{j}\left(f_{i}\right)\right) i^{*}\left(\beta^{l}\left(f_{j}\right)\right)
$$

ce qui prouve la stabilité par composition de $\mathcal{I}_{p}+i^{*} \mathcal{I}_{p}$. Quitte à multiplier par les dénominateurs des $\beta^{l}\left(f_{j}\right)$, l'idéal $\mathcal{I}_{p}+i^{*} \mathcal{I}_{p}$ est inclus dans $\mathcal{O}_{J_{p}^{*}(\Delta)}$. Nous venons de prouver qu'il 
décrit un sous-groupoïde de Lie de $J_{p}^{*}(\Delta)$, la stabilité par composition n'étant vérifiée qu'en dehors du lieu des zéros de ces dénominateurs. Le théorème de prolongement de B. Malgrange [Ma4] assure que l'idéal réduit qu'il engendre différentiablement donne un $\mathcal{D}$-groupoïde de Lie.

Vérifions que ce $\mathcal{D}$-groupoïde de Lie est admissible pour le feuilletage. Les automorphismes locaux du feuilletage qui se factorisent en l'identité sur la transverse agissent comme l'identité sur $\mathcal{O}_{\mathcal{F}}$ donc laissent invariants tous ses idéaux différentiels. Ils sont donc tous solutions du $\mathcal{D}$-groupoïde de Lie que nous venons de construire.

Vérifions enfin que ce $\mathcal{D}$-groupoïde de Lie est propre. On choisit des coordonnées transverses $t$ et des coordonnées tangentes $z$. Les équations d'intégrales premières s'écrivent localement $\frac{\partial H_{i}}{\partial z_{j}}=0$. Les équations supplémentaires de l'idéal premier compatible sont donc des équations que l'on peut supposer, quitte à effectuer les substitutions nécessaires, uniquement en les dérivés des $H_{i}$ par rapport aux $t_{k}$. Cet idéal ne peut être invariant par n'importe quelle transformation en $t$ : le $\mathcal{D}$-groupoïde de Lie construit est donc différent de celui de tous les automorphismes du feuilletage.

Lemme 6.7. Soit $\mathcal{F}$ un germe de feuilletage de codimension un dont le groupoïde de Galois est propre. Il existe une intégrale première de $\mathcal{F}$ dans une extension fortement normale de $\mathcal{M}_{\Delta}$.

Preuve. - En codimension un le rang transverse d'un $\mathcal{D}$-groupoïde de Lie admissible propre est fini. La preuve de ce lemme se fait au cas par cas en discutant suivant le rang transverse du groupoïde de Galois du feuilletage. On sait d'après le théoreme 4.2 qu'il existe dans ces cas des intégrales premières particulières. Ces intégrales premières vont nous donner des idéaux différentiels premiers particuliers de $\mathcal{O}_{\mathcal{F}}$. Les corps des fractions des quotients de $\mathcal{O}_{\mathcal{F}}$ par ces idéaux nous donneront dans chaque cas une extension fortement normale contenant une intégrale première.

\section{Les feuilletages méromorphiquement intégrables.}

Lorsque le rang transverse est nul, le groupoïde de Galois est non transitif et il existe une intégrale première méromorphe. L'extension est $\mathcal{M}_{\Delta}$ et le morphisme est celui qui à $H$ associe une intégrale première méromorphe.

\section{Les feuilletages Darboux-intégrables.}

Lorsque le rang transverse est égal à un, rappelons comment on a construit une intégrale première de type Darboux. Pour $\Gamma \in \operatorname{Aut}\left(\mathcal{F}_{\omega}\right)$, on note $f_{\Gamma}$ la fonction définie par $\Gamma^{*} \omega=$ $f_{\Gamma} \omega$. On notera aussi $\omega=\sum w_{i} d x_{i}$ dans des coordonnées fixées. Le groupoïde de Galois d'un feuilletage Darboux-intégrable est de la forme :

$$
\Gamma^{*} \omega \wedge \omega=0 \text { et } m \circ \Gamma f_{\Gamma}^{k}=m
$$

pour une fonction méromorphe $m$ et un entier $k$. Le système défini par les équations :

$$
(d H)^{\otimes k}-m(x) \omega^{\otimes k}=0
$$

fournit un idéal différentiel premier $\mathcal{J}$ de $\mathcal{O}_{\mathcal{F}}$. Le corps des fractions $\mathcal{K}$ du quotient $\mathcal{O}_{\mathcal{F}} / \mathcal{J}$ est de degré de transcendance un. Pour prouver qu'il s'agit d'une extension fortement normale, prenons $\mathcal{E}$ une extension différentielle de $\mathcal{M}_{\Delta}$ et $\sigma_{1}$ et $\sigma_{2}$ deux plongements de $\mathcal{K}$ dans $\mathcal{E}$. Ces plongements sont complètement déterminés par $H_{1}=\sigma_{1} H$ et $H_{2}=\sigma_{2} H$, où par abus de notation $H$ désigne aussi son image dans $\mathcal{K}$. Les éléments $H_{1}$ et $H_{2}$ de $\mathcal{E}$ vérifient tous les deux l'équation engendrant $\mathcal{J}$. Il existe donc une racine $k$-ième de l'unité $\theta$ et une constante $b$ de $\mathcal{E}$ telles que $H_{1}=\theta H_{2}+b$. Ayant obtenu une expression rationnelle 
de $H_{1}$ en fonction de $H_{2}$ à coefficients dans les constantes de $\mathcal{E}$, on a $\sigma_{1} \mathcal{K} \cdot \mathcal{E}^{c}=\sigma_{2} \mathcal{K} \cdot \mathcal{E}^{c}$. Le corps $\mathcal{K}$ est donc une extension fortement normale de $\mathcal{M}_{\Delta}$ de groupe de Galois les transformations $x \rightarrow \theta x+b$ de la droite affine.

\section{Les feuilletages Liouville-intégrables.}

Lorsque le rang transverse de $\mathcal{F}_{\omega}$ est deux, il existe une forme fermée $\alpha$ vérifiant $d \omega=$ $\omega \wedge \alpha$. Le groupoïde de Galois d'un tel feuilletage a pour équations :

$$
\Gamma^{*} \omega \wedge \omega=0 \text { et } \Gamma^{*} \alpha+\frac{d f_{\Gamma}}{f_{\Gamma}}=\alpha .
$$

On construit des intégrales premières particulières de ce feuilletage en résolvant successivement

$$
\frac{d F}{F}=\alpha \text { puis } d H=F \omega .
$$

Dans des coordonnées on écrit $\omega=\sum w_{i} d x_{i}$ et $\alpha=\sum a_{i} d x_{i}$. Le système d'équations correspondant est :

$$
\frac{\frac{\partial H}{\partial x_{i}}}{w_{i}}=\frac{\frac{\partial H}{\partial x_{j}}}{w_{j}}, \quad \frac{\frac{\partial^{2} H}{\partial x_{i}^{2}}}{\frac{\partial H}{\partial x_{i}}}=a_{i}+\frac{\frac{\partial w_{i}}{\partial x_{i}}}{w_{i}} \text { pour } 0 \leq i, j \leq n .
$$

Ce système donne un idéal différentiel $\mathcal{J}$ de $\mathcal{O}_{\mathcal{F}}$. Le corps des fractions du quotient $\mathcal{K}$ est de degré de transcendance deux. Étant données deux solutions $H_{1}$ et $H_{2}$ des équations ci-dessus dans une extension de $\mathcal{M}_{\Delta}$, on a $H_{1}=a H_{2}+b$ avec $a$ et $b$ deux constantes de l'extension. Le corps $\mathcal{K}$ est donc une extension fortement normale de $\mathcal{M}_{\Delta}$ de groupe de Galois les transformations $x \rightarrow a x+b$ de la droite affine.

\section{Les feuilletages Riccati-intégrables.}

Lorsque le rang transverse de $\mathcal{F}_{\omega}$ est trois, ce feuilletage admet une suite de GodbillonVey de longueur trois : $(\omega, \alpha, \beta)$. On construit des intégrales premières particulières en résolvant la suite d'équations :

$$
\begin{aligned}
d G & =\frac{G^{2}}{2} \omega+G \alpha+\beta \\
\frac{d F}{F} & =G \omega+\alpha \\
d H & =F \omega .
\end{aligned}
$$

Dans des coordonnées on écrit $\omega=\sum w_{i} d x_{i}, \alpha=\sum a_{i} d x_{i}, \beta=\sum b_{i} d x_{i}$. Le système d'équations aux dérivées partielles correspondant est :

$$
\begin{gathered}
\frac{\frac{\partial H}{\partial x_{i}}}{w_{i}}=\frac{\frac{\partial H}{\partial x_{j}}}{w_{j}} \\
\frac{1}{w_{i}}\left(\frac{\frac{\partial^{2} H}{\partial x_{i}^{2}}}{\frac{\partial H}{\partial x_{i}}}-\frac{\frac{\partial w_{i}}{\partial x_{i}}}{w_{i}}-a_{i}\right)=\frac{1}{w_{j}}\left(\frac{\frac{\partial^{2} H}{\partial x_{j}^{2}}}{\frac{\partial H}{\partial x_{j}}}-\frac{\frac{\partial w_{j}}{\partial x_{j}}}{w_{j}}-a_{j}\right) \\
\frac{\partial}{\partial x_{i}}\left(\frac{\frac{\partial^{2} H}{\partial x_{i}^{2}}}{\frac{\partial H}{\partial x_{i}}}\right)-\frac{1}{2}\left(\frac{\frac{\partial^{2} H}{\partial x_{i}^{2}}}{\frac{\partial H}{\partial x_{i}}}\right)^{2}=w_{i}\left(\left(\frac{\frac{\partial w_{i}}{\partial x_{i}}}{w_{i}}\right)-\frac{1}{2}\left(\frac{\frac{\partial w_{i}}{\partial x_{i}}}{w_{i}}\right)^{2}\right) \\
+\frac{\partial a_{i}}{\partial x_{i}}-\frac{1}{2} a_{i}^{2}+b_{i}-a_{i} \frac{\partial w_{i}}{\frac{\partial x_{i}}{w_{i}}} .
\end{gathered}
$$

Ces équations donnent un idéal différentiel $\mathcal{J}$ de $\mathcal{O}_{\mathcal{F}}$. Le corps des fractions du quotient $\mathcal{K}$ est de degré de transcendance trois. Étant données deux solutions $H_{1}$ et $H_{2}$ des 
équations ci-dessus dans une extension de $\mathcal{M}_{\Delta}$ on a $H_{1}=\frac{a H_{2}+b}{c H_{2}+d}$ avec $a, b, c, d$ quatre constantes de l'extension. Le corps $\mathcal{K}$ est donc une extension fortement normale de $\mathcal{M}_{\Delta}$ de groupe de Galois les transformations homographiques de la droite projective.

\section{RÉFÉRENCES}

[Bu1] A. Buium - Differential function fields and moduli of algebraic varieties, Lecture Notes in Mathematics 1226, Springer-Verlag (1986)

[Bu2] A. Buium - Differential algebra and diophantine geometry, Actualités Mathématiques, Hermann (1994)

[B-T] M. Berthier et F. Touzet - Sur l'intégration des équations différentielles holomorphes réduites en dimension 2, Boletim da Soc. Bra. Mat. vol 30, $n^{\circ} \mathbf{3}$ (1999)

[Cas1] G. Casale - Suites de Godbillon-Vey et intégrales premières, C. R. Acad. Sci. Paris 335 (2002)

[Cas2] G. Casale - D-enveloppe d'un difféomorphisme de $(\mathbb{C}, 0)$ "Proceedings of the conference Resurgence, Alien Calculus, Resummability, Transseries" Ann. Fac. Sci. Toulouse Math. (6) 13 No4 (2004)

[CLLPT] D. Cerveau, A. Lins Neto, F. Loray, J.V. Pereira, F. Touzet - Algebraic Reduction Theorem for complex codimension one singular foliations http ://arxiv.org/PS_cache/math/pdf/0406/0406293.pdf

[Dum] S. Dumitrescu - Structures géometriques holomorphes sur les variétés complexes compactes, Ann. Sci. Écoles Normale Sup., $4^{e}$ série, t. 34 (2001)

[Dr1] J. Drach - Essai sur une théorie générale de l'intégration et sur la classification des transcendantes, Ann. Sci. Écoles Normale Sup. (1898)

[Dr2] J. Drach - Sur le problème logique de l'intégration des équations différentielles, Ann. Fac. Sci. de l'Université de Toulouse (1908)

[Ec] J. Écalle - Les fonctions résurgentes Tomes 1 et 2, Publication mathématiques d'Orsay (1981)

[Ei] D. Eisenbud - Commutative Algebra with a View Toward Algebraic Geometry, Graduate Text in Mathematics 150 Springer-Verlag (1995)

[Fr] J. Frisch - Points de platitude d'un morphisme d'espaces analytiques complexes, Invent. Math. 4 (1967)

[Go] C. Godbillon - Feuilletage. Études géométriques, Progress in Mathematics 98 (1991)

[G-V] C .Godbillon et J. Vey - Un invariant des feuilletages de codimension un, C. R. Acad. Sci. Paris 273 (1971)

[Gr] M. Gromov - Rigid transformation groups, Géométrie Différentielle, Tavaux en cours, Hermann, 33 (1988)

[II] Y.S. Ilyashenko - Divergence of series that reduce an analytic differential equation to linear normal form at a singular point, (Russian) Funktsional. Anal. i Prilozhen. $13 n^{\circ} \mathbf{3}$ (1979)

[Ko] E.R. Kolchin - Differential Algebra and Algebraic Groups, Pure and Applied Mathematics, Vol. 54. Academic Press, (1973)

[Kov] J.J. Kovacic - The differential Galois theory of strongly normal extensions, Trans. Amer. Math. Soc. 355 (2003)

[K-S] A. Kumpera et D. Spencer - Lie Equations, Ann. of Math. Studies, Princeton Univ. Press (1972)

[Lie] S. Lie - Transformationgruppen Tome 3, Chelsea Publishing Co., (1970).

[Mck] K. Mackenzie - Lie groupoids and Lie algebroids in differential geometry, L.N.S. 124 Cambridge Univ. Press Cambridge (1987)

[Ma1] B. Malgrange - Travaux d'Écalle et Martinet-Ramis sur les systèmes dynamiques, Séminaire Bourbaki vol 1981/82 Astérisque 92-93 Soc. Math. France (1982) 
[Ma2] B. Malgrange - L'involutivité générique des systèmes différentiels analytiques, C. R. Acad. Sci. Paris 326 (1998)

[Ma3] B. Malgrange - Germes de $\mathcal{D}$-groupoïdes en dimension un, (notes informelles) (2000)

[Ma4] B. Malgrange - Le groupoïde de Galois d'un feuilletage, Monographie $\mathbf{3 8}$ vol 2 de L'enseignement mathématique (2001)

[Ma5] B. Malgrange - On the non linear Galois differential theory, Chinese Ann. Math. Ser. B 23 $n^{\circ} \mathbf{2},(2002)$

[Ma6] B. Malgrange - Systèmes Différentiels Involutifs, Prépublication de l'Institut Fourier $n^{\circ} \mathbf{6 3 6}$, (2004)

[M-M] J.F. Mattei et R. Moussu - Holonomie et intégrale première, Ann. Sci. École Normale Sup. 13 (1980)

[M-R1] J. Martinet et J.P. Ramis - Problèmes de modules pour les équations différentielles non linéaires du premier ordre., Inst. Hautes études Sci. Publ. Math. 55 (1982)

[M-R2] J. Martinet et J.P. Ramis - Classification analytique des équations différentielles non linéaires résonnantes du premier ordre., Ann. Sci. École Normale Sup. 16 (1983)

[Po] J.F. Pommaret - Differential Galois Theory, Mathematics and its Applications, 15. Gordon \& Breach Science Publishers, New York (1983)

[P-S] M.J. Prelle et M.F. Singer - Elementary first integrals of differential equations, Trans. Amer. Math. Soc. 279 (1983)

[Ra] J.P. Ramis - Séries Divergentes et Théories Asymptotiques,Panorama et Synthèse 1 S.M.F. (1993)

[Ri2] J.F. Ritt - Differential Algebra, American Mathematical Society Colloquium Publications, Vol. XXXIII, American Mathematical Society, New York (1950)

[Sc] B.A. Scàrdua - Transversely affine and transversely projective holomorphic foliations, Ann. Sci. École Norm. Sup. 30 (1997)

[Se] A. Seidenberg - Reduction of singularities of the differential equation $A d y=B d x$, Amer. J. Math. 90 (1968)

[Si] M. Singer - Liouvillian first integral of differential equations, Trans. A. M. S. 333 (1992)

[St] S. Sternberg - Lectures on differential geometry, Second edition. Chelsea Publishing Co. (1983)

[To] J.C. Tougeron - Idéaux de fonctions différentiables, Ergebnisse der Mathematik und ihrer Grenzgebiete, 71, Springer-Verlag (1972)

[Tou1] F. Touzet - Equation différentielles admettant des solutions liouvilliennes, thèse de l'Université de Rennes I (1995)

[Tou2] F. Touzet - Sur les feuilletages holomorphes transversalement projectifs, Ann. Inst. Fourier, Grenoble 53, 3 (2003)

[Ves1] E. Vessiot - Sur une théorie générale de la réductibilité des équations et systèmes d'équations finies ou différentielles, Ann. Sci. École Normale Sup. 63 (1946)

[Ves2] E. Vessiot - Sur la réductibilité des équations aux dérivées partielles du $1^{\mathrm{er}}$ ordre, à une inconnue, qui ne la contiennent pas et sont linéaires et homogènes par rapport à ses dérivées, Bull. Soc. Math. France 75 (1947)

Laboratoire Émile Picard, umr 5580 ufr mig, Université Paul Sabatier, 118 Route de Narbonne, 31062 Toulouse Cedex 4, France

E-mail address: casale@picard.ups-tlse.fr 